Huchon, P., Taylor, B., and Klaus, A. (Eds.)

Proceedings of the Ocean Drilling Program, Scientific Results Volume 180

\section{QUATERNARY RADIOLARIANS IN THE WESTERN WOODLARK BASIN, SOUTHWEST PACIFIC (ODP LEG 180)'}

\author{
Massimiliano Testa, ${ }^{2}$ Giuseppe Cortese, ${ }^{3}$ Stefania Gerbaudo, ${ }^{2}$ and \\ Eugenio Andri ${ }^{2}$
}

\begin{abstract}
Cores from the 11 sites drilled during Leg 180 showed radiolarian assemblages that appear only in the Quaternary sediments. The most diverse and well-preserved assemblages were found in hemipelagic sediments from Holes 1108A, 1110A, and 1115B.

\section{INTRODUCTION}

During Ocean Drilling Program (ODP) Leg 180, a transect of 11 sites was drilled in the Western Woodlark Basin (Table T1). The age of the recovered sediments ranges from Miocene to Pleistocene, and radiolarians were found only in a few samples of Pleistocene age. The assemblage consists of 121 taxa. Often the preservation is not very good, as evidenced by thinner tests. Although the data obtained during this study do not allow high stratigraphic resolution, they are nonetheless sufficient to assign the studied sediments to the Pleistocene.

\section{MATERIALS AND METHODS}

A preliminary study was carried out on more than 100 sediment samples to verify the presence of radiolarian assemblages and to give a stratigraphic interpretation of the cored sediments.
\end{abstract}

T1. Geographic location and water depths of Leg 180 holes, p. 13.

${ }^{1}$ Testa, M., Cortese, G., Gerbaudo, S., and Andri, E., 2001. Quaternary radiolarians in the western Woodlark Basin, southwest Pacific (ODP Leg 180). In Huchon, P., Taylor, B., and Klaus, A. (Eds.), Proc. ODP, Sci. Results, 180, 1-21 [Online]. Available from World Wide Web: <http://www-odp. tamu.edu/publications/180_SR/ VOLUME/CHAPTERS/170.PDF>. [Cited YYYY-MM-DD]

${ }^{2}$ Dipartimento per lo Studio del Territorio e delle sue Risorse, Università degli Studi di Genova, Corso Europa, 26-16132 Genova, Italy. Correspondence author: gerbaudo@box.tin.it; gerbaudo@dipteris.unige.it; and gerbaudo@noos.fr

${ }^{3}$ Alfred Wegener Institute for Polar and Marine Research (AWI), Columbusstrasse, PO Box 120161, 27515 Bremerhaven, Federal Republic of Germany.

Initial receipt: 12 December 2000 Acceptance: 19 April 2001

Web publication: 14 August 2001

Ms 180SR-170 
Material from all sites, with the exception of Sites 1113 and 1117, and from all the encountered nonmarine sedimentary facies has been sampled. Sites 1113 and 1117 were not analyzed because they were lacking in sediments.

The result of a pilot study showed that radiolarians were confined exclusively to sediments of Quaternary age. A more detailed study was then carried out on the Quaternary sediments recovered from Holes 1108B, 1109C, 1110A, 1111A, 1112A, 1114A, 1115A, and 1115B. We typically analyzed two samples per core, with a total of 78 studied samples (Table T2).

Radiolarian assemblages, generally poorly preserved, were recovered in only 19 of these samples (Table T3). The general morphology of the specimens and their taxonomic identification were determined using a transmitted light microscope, whereas finer details of the test surface were analyzed by means of a scanning electron microscope.

Samples were prepared from core material according to the following procedure:

1. The sediment was dried in an oven for $5 \mathrm{hr}$ at $100^{\circ} \mathrm{C}$.

2. $10 \mathrm{~g}$ of dried sediment was placed in a beaker and boiled for 12 hr in diluted (5 parts water to 1 part peroxide) hydrogen peroxide.

3. Diluted (15\%) hydrochloric acid $(\mathrm{HCl})$ was added to the sample after it had cooled to room temperature.

4. The residue was sieved ( $45-\mu \mathrm{m}$ mesh size).

5. The remaining residue was mixed in a beaker with $100 \mathrm{~mL}$ of distilled water and put into suspension. A fixed split $(100 \mathrm{~mL})$ of this suspension was taken by a pipette and strewn on a $27 \mathrm{~mm} \times$ $46 \mathrm{~mm}$ coverslip.

6. The coverslip was dried on an hot plate at moderate temperature $\left(30^{\circ}-40^{\circ} \mathrm{C}\right)$ and then fixed to a slide with glycerine.

Because many of the studied samples showed a relevant clay component, the hydrogen peroxide boiling was repeated several times until the radiolarian tests were clean and free of any clay coating.

In some of the most clay-rich samples, dry sodium hexametaphosphate was added to the hydrogen peroxide solution to increase the effectiveness of clay mineral breakdown.

\section{RADIOLARIAN PRESERVATION}

Dissolution phenomena resulting in thinning of the radiolarian tests are linked to the various sediments containing them and sometimes to the presence of ash-rich layers.

In particular, the assemblages found in hemipelagic sediments mainly made up of clay or silty clay-bearing nannofossil ooze at the top of Holes 1108B, 1110A (Sample 180-1110A-1H-2, 77-79 cm), and 1115B (Sample 180-1115B-1H-1, 5-7 cm) appear to be the best preserved and the richest in specimens. In some sediments consisting of volcanic ash layers (Sample 180-1109C-3H-1, 49-50 cm), silty clay with volcanic ash (Sample 180-1109C-4H-1, 58-60 cm), and silty clay (Sample 180$1109 \mathrm{C}-6 \mathrm{H}-5,24-26 \mathrm{~cm}$ ), very few and poorly preserved specimens were found.
T2. Full designation of studied samples, p. 14.

T3. Distribution of radiolarian taxa, p. 15. 
In samples made up mainly of silty clay in Holes $1111 \mathrm{~A}$ and $1112 \mathrm{~A}$, the assemblages show an intermediate state of preservation compared to those described above.

In conclusion, we observed that usually under the same textural conditions in samples where ash layers are present (i.e., Sample 180-1115B$2 \mathrm{H}-4,145-147 \mathrm{~cm})$, test preservation is poor.

\section{QUATERNARY RADIOLARIAN ZONATION}

The radiolarian zonal scheme and code numbers adopted in this study (Sanfilippo and Nigrini, 1998) have been integrated with other published radiolarian zonations and their chronological interpretation (Nigrini, 1971; Caulet et al., 1993; Shackleton et al., 1995).

The Pleistocene epoch has been subdivided into five radiolarian biozones (Sanfilippo and Nigrini, 1998):

RN17: Buccinosphaera invaginata Taxon-Range Zone (Nigrini, 1971), defined by the range of $B$. invaginata.

RN16: Collosphaera tuberosa Interval Zone (Nigrini, 1971; emend. Caulet, 1979). The top is defined by the first morphotypic occurrence (FO) of $B$. invaginata and the base by the last morphotypic occurrence (LO) of Stylatractus universus.

RN15: S. universus Concurrent Range Zone (Caulet,1979; renamed by Johnson et al., 1989). The top is defined by the LO of $S$. universus and the base by the FO of C. tuberosa.

RN14: Amphirhopalum ypsilon Interval Zone (Nigrini, 1971). The top is defined by the FO of C. tuberosa and the base by the LO of Anthocyrtidium angulare.

RN13: A. angulare Interval Zone (Nigrini, 1971). The top is defined by the LO of A. angulare and the base by the LO of Pterocanium prismatium.

\section{LEG 180 RADIOLARIAN BIOSTRATIGRAPHY}

Zonal assignments for each of the studied samples is reported in Table T4.

Below, we discuss the occurrence and/or the absence of radiolarian species that can be used as stratigraphic markers in each of the studied samples. However, our sampling spacing is too broad to carry out a detailed stratigraphic study. Moreover, the paucity of radiolarian specimens in some of the samples could lead to the wrong inferences when interpreting the absence of a certain stratigraphically relevant taxon, as many of these taxa are rare even in well-preserved material.

\section{Hole 1108B}

Sample 180-1108B-1R-1, 10-12 cm, contains C. tuberosa, which constrains the age of this sample to the late Quaternary (S. universus Zone and younger).

The occurrence of $C$. tuberosa and the absence of both $B$. invaginata and $S$. universus would place this sample in the $C$. tuberosa Zone RN16 itself, spanning from 0.42 to $0.18 \mathrm{Ma}$ (Johnson et al., 1989). However, because of the sparseness of the assemblage, a broader, late Quaternary age determination is suggested.
T4. Radiolarian biozone assignments, p. 18. 
The scant assemblage contained in the lower sample of this core (Sample 180-1108B-1R-2, 50-52 cm) and the absence of any stratigraphic markers hindered the possibility to assign it to any radiolarian zone.

\section{Hole 1109C}

The only three samples containing radiolarians have abundances that are too scarce to allow for any zonal assignment.

\section{Hole 1110A}

The presence of C. tuberosa in Sample 180-1110A-2H-1, 58-62 cm, and the absence of $B$. invaginata and $S$. universus from Samples $1 \mathrm{H}-2$, $77-79 \mathrm{~cm}$, and $2 \mathrm{H}-1,58-62 \mathrm{~cm}$, leads us to assign these samples to Zone RN16 (see also "Hole 1108B," p. 3). As for Sample 180-1110A-2H-1, 81$85 \mathrm{~cm}$, there are no data available for any zonal assignment.

\section{Hole 1111 A}

The presence of Theocorythium trachelium in all but one of the studied samples indicates a Quaternary age for all of them. In fact, this taxon is found in variable abundances at the base of the Quaternary in the tropical Pacific, whereas it is essentially absent at the top of the Pliocene (Nigrini, 1971).

More recently, Shackleton et al. (1995) assigned a 1.55-Ma age to the first appearance of T. trachelium.

The tests of A. ypsilon present in Samples 180-1111A-14R-1, 60-62 $\mathrm{cm}$, and 14R-3, 62-64 cm, are characterized by the presence of four to five chambers before the branching of the arms. These morphotypes have been considered by Nigrini (1971) as being typical for the late Quaternary.

Based on this consideration, we assign a late Quaternary age to the latter two samples.

\section{Hole 1112A}

The only stratigraphically significant species recognized at this site is Lamprocyrtis nigriniae.

As Shackleton et al. (1995) date the first appearance of $L$. nigriniae at 1.23 Ma, the presence of this taxon indicates a Quaternary age for the only sample in which radiolarians were present (Sample 180-1112A-1R$1,100-101 \mathrm{~cm})$.

\section{Hole 1115A}

No radiolarian tests were observed in any of the studied samples.

\section{Hole 1115B}

T. trachelium was found in the two upper samples (Samples 180$1115 \mathrm{~B}-1 \mathrm{H}-1,5-7 \mathrm{~cm}$, and $2 \mathrm{H}-4,145-147 \mathrm{~cm})$, therefore indicating a Pliocene or a Quaternary age.

A. ypsilon was also found in Sample 180-1115B-1H-1, 5-7 cm, narrowing its age to the late Quaternary (see "Hole 1111A," p. 4). This conclusion is also supported by the presence of Pterocorys hertwigii in 
the same sample, as the FO of this taxon takes place in the A. ypsilon Zone (Sanfilippo and Nigrini, 1998).

The presence of A. ypsilon in Sample 180-1115B-3H-2, 58-60 cm, indicates an age ranging from early Pliocene to present, as this species has its first occurrence within the Phormostichoartus doliolum Zone (Sanfilippo and Nigrini, 1998) and is still extant.

No stratigraphically significant taxa have been observed in Samples 180-1115B-4H-6, 66-68 cm, and 5H-1, 19-21 cm.

\section{RADIOLARIAN FAUNAL LIST}

An alphabetically sorted list of the radiolarian taxa recognized in this study follows.

Additional pictures and synonymies for most of the taxa in the following list are reported in Nigrini $(1967,1968,1970,1977)$, Goll (1968, 1969, 1976), Riedel and Sanfilippo (1971, 1978), Nigrini and Moore (1979), and Boltovskoy and Riedel (1980).

Given the less-than-optimal preservation of the studied material, some of the observed taxa were classified only at genus or family level.

Acanthosphaera actinota (Haeckel, 1860b)

Acrosphaera spinosa (Haeckel, 1860a) (Pl. P1, fig. 1)

Actinomma spp.

Actinomma boreale (Cleve, 1899)

Actinomma haysi Bjøklund, 1976

Actinomma leptoderma (Jørgensen, 1900)

Remarks: Synonyms and additional taxonomic information in Cortese and Bjørklund (1998b)

Actinomma sol Cleve, 1899

Remarks: See also Boltovskoy and Riedel (1980).

Amphirhopalum ypsilon Haeckel, 1887 (Pl. P1, fig. 2)

Amphisphaera spp.

Anthocyrtidium ophirense (Ehrenberg, 1872)

Anthocyrtidium zanguebaricum (Ehrenberg, 1872)

Arachnocorys umbellifera (Haeckel, 1887)

Artostrobus annulatus (Bailey, 1856)

Artostrobus joergenseni Petrushevskaya, 1971

Botryocyrtis scutum (Harting, 1863) (Pl. P1, fig. 3)

Botryopyle cribrosa (Popofsky, 1913)

Botryostrobus aquilonaris (Bailey, 1856)

Botryostrobus auritus/australis (Ehrenberg, 1844)

Callimitra spp.

Carpocanarium papillosum (Ehrenberg, 1872)

Carpocanistrum spp.

Remarks: For a definition of this morphotype see Riedel and Sanfilippo (1971); Nigrini and Moore (1979).

Cladoscenium spp.
P1. Radiolarians from Leg 180, p. 19.

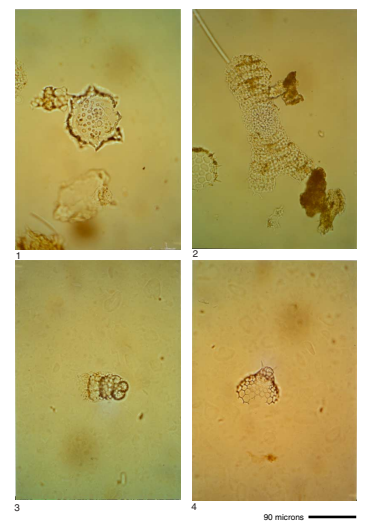




\section{TESTA ET AL. \\ QUATERNARY RADIOLARIANS}

Clathrocanium coarctatum Ehrenberg, 1860

Clathrocanium reginae Haeckel, 1887

Collosphaera spp.

Collosphaera macropora Popofsky, 1917

Collosphaera tuberosa Haeckel, 1887

Cornutella profunda Ehrenberg, 1858 (P1. P1, fig. 5)

Cycladophora bicornis (Popofsky, 1908) (Pl. P1, fig. 4)

Cycladophora davisiana (Ehrenberg) cornutoides (Petrushevskaya, 1967)

Cycladophora davisiana (Ehrenberg) davisiana (Petrushevskaya, 1967).

Cyrtolagena laguncula Haeckel, 1887

Dictyocoryne profunda Ehrenberg, 1860

Dictyocoryne truncatum (Ehrenberg, 1861)

Remarks: Transitional forms between Dictyocoryne truncatum and Euchitonia spp. have been observed and counted as Dictyocoryne truncatum. Further details in Van de Paverd (1995).

Dictyophimus spp.

Dictyophimuscrisiae Ehrenberg, 1854

Remarks: Dictyophimus hirundo (Haeckel, 1887) has also been included.

Didymocyrtis tetrathalamus (Haeckel, 1887)

Dorydruppa bensoni Takahashi, 1991

Druppatractus irregularis (Bailey, 1856)

Eucecryphalus gegenbauri Haeckel, 1860a

Euchitonia spp.

Remarks: This taxon includes those specimens lacking complete arms, which were therefore not possible to identify at a species level.

Euchitonia elegans (Ehrenberg, 1872)

Euchitonia furcata Ehrenberg, 1872 (Pl. P1, fig. 6)

Eucyrtidium spp.

Eucyrtidium acuminatum (Ehrenberg, 1844)

Eucyrtidium anomalum (Haeckel, 1860a) (P1. P1, fig. 7)

Eucyrtidium hexagonatum Haeckel, 1887

Eucyrtidium teuscheri Haeckel teuscheri Caulet, 1986

Giraffospyris angulata (Haeckel, 1887)

Heliodiscus asteriscus Haeckel, 1887

Hexacontium spp.

Hexacontium enthacanthum Jørgensen, 1900

Hexacontium laevigatum Haeckel, 1887

Hexacontium pachydermum Jørgensen, 1900

Remarks: This genus was emended by Cortese and Bjørklund (1998a). In Sample $180-1112 \mathrm{~A}-1 \mathrm{R}-1,100-101 \mathrm{~cm}$, the three specimens assigned to this species had a cortical shell diameter $>130 \mu \mathrm{m}$, therefore bigger than the typical H. pachydermum as defined by Jørgensen. They could therefore represent a different low-latitude morphotype. We, however, include them in this taxon, as all the other taxonomic characters coincide. 


\section{Testa et Al. \\ QUATERNARY RADIOLARIANS}

Hexacontium pythagoraea (Haeckel, 1887)

Hexapyle dodecantha Haeckel, 1887

Hexastylus spp.

Hymeniastrum euclidis (Haeckel, 1887)

Lamprocyclas maritalis maritalis Haeckel, 1887 (Pl. P1, fig. 8)

Lamprocyrtis nigriniae (Caulet, 1971)

Larcoidea spp.

Larcopyle bütschlii Dreyer, 1889

Larcospira quadrangula Haeckel, 1887

Liriospyris reticulata (Ehrenberg, 1872)

Litharachnium tentorium Haeckel, 1860a

Lithelius minor Jørgensen, 1899

Lithobotrys inflatum Bailey, 1856

Lithocampe furcaspiculata Popofsky, 1908

Lithocampe platycephala (Ehrenberg, 1872)

Lithomelissa setosa Jørgensen, 1900

Lithomelissa thoracites Haeckel, 1862

Lophophaena bütschlii (Haeckel, 1887)

Lophophaena hispida (Ehrenberg, 1872)

Lophospyris pentagona pentagona (Ehrenberg, 1872)

Otosphaera polymorpha Haeckel, 1887

Peridium longispinum Jørgensen, 1900

Peripyramis circumtexta Haeckel, 1887

Peromelissa phalacra Haeckel, 1887

Phormospyris stabilis scaphipes (Haeckel, 1887)

Phormospyris stabilis stabilis (Goll, 1968)

Phormostichoartus corbula (Harting, 1863)

Porodiscus sp.

Remarks: similar to Porodiscus sp. A in Nigrini and Moore (1979).

Pseudodictyophimus gracilipes (Bailey, 1856)

Pterocanium praetextum (Ehrenberg, 1872) eucolpum (Haeckel, 1887)

Pterocanium praetextum praetextum Ehrenberg (1872) (P1. P1, fig. 9)

Pterocanium spp.

Pterocanium trilobum (Haeckel, 1860a)

Pterocorys clausus (Popofsky, 1913)

Remarks: Pterocorys zancleus (Müller) has also been included.

Pterocorys hertwigii (Haeckel, 1887)

Pterocorys minythorax (Nigrini, 1968)

Pylospira octopyle (Haeckel, 1887

Rhizoplegma boreale (Cleve, 1899) 


\section{Testa et Al. \\ QUATERNARY RADIOLARIANS}

Siphocampe arachnea (Ehrenberg, 1838)

Siphocampe lineata (Ehrenberg, 1838)

Siphocampe nodosaria (Haeckel, 1887)

Siphonosphaera polysiphonia Haeckel, 1887

Spongaster tetras tetras Ehrenberg, 1860 (Pl. P1, fig. 10)

Spongocore puella Haeckel, 1887 (Pl. P1, fig. 11)

Spongodiscus resurgens Ehrenberg, 1854

Spongopyle osculosa Dreyer, 1889 (Pl. P1, fig. 12)

Spongotrochus glacialis Popofsky, 1908

Spongurus(?) sp. Petrushevskaya, 1967

Remarks: For descriptions and pictures see Petrushevskaya (1967) and Ling et al. (1971).

Songurus ellipticus (Ehrenberg, 1872)

Spongurus pylomaticus Riedel, 1958

Spirocyrtis scalaris Haeckel, 1887

Stylatractus spp.

Remarks: Described by Nigrini and Moore (1979) as Stylatractus sp. and by Boltovskoy (1998) as Stylatractus spp. group.

Stylochlamydium asteriscus Haeckel, 1887

Stylochlamydium venustum (Bailey, 1856)

Stylodictya aculeata Jørgensen, 1905

Stylodictya validispina Jørgensen, 1905

Tessarastrum straussi Haeckel, 1862

Tetrapyle octacantha Müller, 1858

Remarks: Octopyle stenozona Haeckel has also been included.

Theocorys veneris Haeckel, 1887

Theocorythium trachelium (Ehrenberg, 1872)

Theopylium tricostatum Haeckel, 1887

Tholospyris tripodiscus Haeckel, 1887

Trissocyclidae spp.

Remarks: This taxon includes all nassellarians having a sagittal ring and not included in any other species-level taxon.

Zygocircus productus (Hertwig, 1879)

Remarks: Tholospyris sp. Takahashi and Honjo (1981) has also been included.

\section{CONCLUSIONS}

Radiolarian assemblages are mainly localized in the Quaternary upper portions of the recovered cores at Sites 1108, 1109, 1110, 1111, 1112 , and 1115.

Considering the less-than-optimal preservation for many of the samples studied, we cannot perform a detailed stratigraphic zonation. With regard to the poor test preservation, even if we believe that it is due to secondary dissolution processes considering the uniformity of the phenomenon on the entire test surface, we could even suppose that the test 
M. TESTA ET AL.

QUATERNARY RADIOLARIANS

thinness is original and due to silica scarcity in the living environment, as we observe at present in tropical areas.

We also point out that the best-preserved specimens were recovered in hemipelagic sediments rich in calcareous nannoplankton; this could lead one to suppose a preserving action linked to sedimentation velocity and to "proofing" induced by these sediments.

\section{ACKNOWLEDGMENTS}

SG thanks the Italian Secretariat for financial support to participate in Leg 180 and carry out this research. This work was also supported by the funds to E. Andri (University of Genova). Thanks also go to A. Sanfilippo and the ODP editorial staff for their reviews of this paper and to R. Laronga, who corrected our English. 


\section{REFERENCES}

Bailey, J.W., 1856. Notice of microscopic forms found in the soundings of the Sea of Kamtschatka-with a plate. Am. J. Sci. Arts, Ser. 2, 64:1-6.

Bjørklund, K.R., 1976. Radiolarians from the Norwegian Sea, Leg 38 of the Deep Sea Drilling Project. In Talwani M., Udinitsev, G., White, S.M., Init. Rept. DSDP 38: Washington (U.S. Govt. Printing Office), 1101-1168.

Boltovskoy, D., 1998. Classification and distribution of South Atlantic Recent polycystine Radiolaria. Paleontologia Electronica, 1:1-116 [Online]. Available from the Word Wide Web: http://www.erdw.ethz.ch/ pe/1998_2/toc.htm. [2000-01-03]

Boltovskoy, D., and Riedel, W.R., 1980. Polycystine radiolaria from the southwestern Atlantic Ocean plankton. Rev. Esp. Micropaleontol., 12:99-146.

Caulet, J.-P., 1971. Contribution a l'étude de quelques Radiolaries Nassellaires des boues de la Méditerranée et du Pacifique (Study of some nassellarian Radiolaria from Mediterranean and Pacific sediments). Archives originale, Centre de Documentation, C.N.R.S., No. 498. Cah. Micropaleontol., Ser. 2, 10:1-10.

- 1979. Les depots a radiolaires d'age Pliocene superieur a Pleistocene dans l'ocean Indien central: nouvelle zonation biostratigraphique (Radiolarian upper Pliocene-Pleistocene deposits in the central Indian Ocean; new biostratigraphic zonation). Mem. Mus. Nat. Hist. Nat. Ser. C, 43:119-141.

- 1986. Radiolarians from the southwest Pacific. In Kennett, J.P., von der Borch, C.C., et al., Init. Repts. DSDP, 90: Washington (U.S. Govt. Printing Office), 835-861.

Caulet, J.-P., Nigrini, C., and Schneider, D.A., 1993. High resolution Pliocene-Pleistocene radiolarian stratigraphy of the tropical Indian Ocean. Mar. Micropaleontol., 22:111-129.

Cleve, P.T., 1899. Plankton collected by the Swedish expedition to Spitzbergen. Kongl. Sven. Vetensk.-Akad. Handl., 32:2-34.

Cortese G., and Bjørklund, K.R., 1998a. Morphometry and taxonomy of Hexacontium species from western Norwegian fjords. Micropaleontology, 44:161-172.

- 1998b. The taxonomy of boreal Atlantic Ocean Actinommidae (radiolaria). Micropaleontology, 44:146-160.

Dreyer, F., 1889. Morphologische Radiolarienstudien. 1. Die Pylombildungen in vergleichend-anatomischer und entwicklungsgeschichtlicher Beziehung bei Radiolarien und bei Protisten überhaupt, nebst System und Beschreibung neuer und der bis jetzt bekannten pylomatischen Spumellarien. Jena. Z. Naturwiss., 23:1-138.

Ehrenberg, C.G., 1838. Über die Bildung der Kreidefelsen und des Kreidemergels durch unsichtbare Organismen. K. Akad. Wiss. Berlin, Abh. 1838 (1840, separate 1839): 59-148.

—, 1844. Über 2 neue Lager von Gebirgsmassen aus Infusorien als Meeres-Absatz in Nord-Amerika und eine Vergleichung derselben mit den organischen Kreide-Gebilden in Europa und Afrika. K. Preuss. Akad. Wiss. Berlin, Berichte, 57-97.

- 1854. Mikrogeologie: Das Erden und Felsen schaffende Wirken des unsichtbar kleinen selbständigen Lebens auf der Erde: Leipzig (Leopold Voss).

- 1858 . Kurze Characteristik der 9 neuen Genera und der 105 neuen Species des agaischen Meeres und des Tiefgrundes des Mittel-Meeres. K. Preuss. Akad. Wiss. Berlin, Monatsberichte, 1858:10-40.

— 1860 . Über die organischen und unorganischen Mischungsverhaltnisse des Meeresgrundes in 19,800 Fuss Tiefe nach Lieut. Brookes Messung. K. Preuss. Akad. Wiss. Berlin, Monatsberichte, Jahre 1860:765-774.

_ 1861 . Über die Tiefgrund-Verhältnisse des Oceans am Eingange der Davisstrasse und bei Island. K. Preuss. Akad. Wiss. Berlin, Monatsberichte, 1861:275-315. 
1872. Mikrogeologische Studien als Zusammenfassung der Beobachtungen des kleinsten Lebens der Meeres-Tiefgrunde aller Zonen und dessen geologischen Einfluss. K. Preuss. Akad. Wiss. Berlin, Monatsberichte, 265-322.

Goll, R.M., 1968. Classification and phylogeny of Cenozoic Trissocyclidae (Radiolaria) in the Pacific and Caribbean basins, Part I. J. Paleontol., 42:1409-1432.

, 1969. Classification and phylogeny of Cenozoic Trissocyclidae (Radiolaria) in the Pacific and Caribbean basins, Part II. J. Paleontol., 43:322-339.

- 1976. Morphological intergradation between modern populations of Lophospyris and Phormospyris (Trissocyclidae, Radiolaria). Micropaleontology, 22:379_ 418.

Haeckel, E., 1860a. Fernere Abbildungen und Diagnosen neuer Gattungen und Arten von lebenden Radiolarien des Mittelmeeres (Supplementary illustrations and diagnosis of new genera and species of living radiolarians of the Mediterranean Sea). $K$. Preuss. Akad. Wiss. Berlin, Monatsberichte, 835-845.

— $1860 \mathrm{~b}$. Über neue, lebende Radiolarien des Mittelmeeres und... die dau gehorigen Abbildungen (Some new living Radiolaria from the Mediterranean Sea and ... their illustration). K. Preuss. Akad. Wiss. Berlin, Monatsberichte, 794-817.

—, 1862. Die Radiolarien (Rhizopoda Radiolaria): Berlin (Reimer).

, 1887. Report on the Radiolaria collected by H.M.S. Challenger during the years 1873-1876. Rep. Sci. Results Voy. H.M.S. Challenger, 1873-1876, Zool., 1887:11803.

Harting, P., 1863. Bijdrage tot de kennis der mikroskopische faune en flora van de Banda-Zee [Contribution to the knowledge of microscopic fauna and flora from the Banda Sea]. Kon. Akad. Wetensch. Amsterdam, Verh., 10:1-34.

Hertwig, R., 1879. Der Organismus der Radiolarien. Jenaische Denkshr., 2:129-277.

Johnson, D.A., Schneider, D.A., Nigrini, C.A., Caulet, J.-P., and Kent, D.V., 1989. Pliocene-Pleistocene radiolarian events and magnetostratigraphic calibrations for the tropical Indian Ocean. Mar. Micropaleontol., 14:33-66.

Jørgensen, E., 1899. Protophyten und Protozoen im Plankton aus der Norwegischen Westkuste. Bergens Mus. Aarbog, 6:1-112.

- 1900. Protophyten und Protozoen im Plankton aus der norwegischen Westkuste. Bergens Mus. Aarbog (1899), 2:1-112.

- 1905. The protist plankton and the diatoms in bottom samples. Bergens Mus. Skr., 49-151.

Ling, H.Y., Stadum, C.J., and Welch, M.L., 1971. Polycystine Radiolaria from Bering Sea surface sediments. In Farinacci, A. (Ed.), Proc. 2nd Planktonic Conf., Roma. Rome (Ed. Technosci.), 705-729.

Müller, J., 1858. Über die Thalassicollen, Polycystinen und Acanthometren des Mittelmeeres. K. Preuss. Akad. Wiss. Berlin, Abh., 1-62.

Nigrini, C., 1967. Radiolaria in pelagic sediments from the Indian and Atlantic Oceans. Bull. Scripps Inst. Oceanogr., 11:1-125.

- 1968. Radiolaria from eastern tropical Pacific sediments. Micropaleontology, 14:51-63.

- 1970. Radiolarian assemblages in the North Pacific and their application to a study of Quaternary sediments in core V20-130. In Hays, J.D. (Ed.), Geological Investigations of the North Pacific. Mem.-Geol. Soc. Am., 126:139-183.

- 1971. Radiolarian zones in the Quaternary of the equatorial Pacific Ocean. In Funnell, B.M., and Riedel, W.R. (Eds.), The Micropalaeontology of Oceans: Cambridge (Cambridge Univ. Press), 443-461.

- 1977. Tropical Cenozoic Artostrobiidae (Radiolaria). Micropaleontology, 23:241-269.

Nigrini, C., and Moore, T.C., 1979. A Guide to Modern Radiolaria. Spec. Publ. Cushman Found. Foraminiferal Res., 16.

Petrushevskaya, M.G., 1967. Radiolarians of the orders Spumellaria and Nassellaria of the Antarctic region. In Andriiashev, A. P., and Ushako, P. V. (Eds.), Biological 
Reports of the Soviet Antarctic Expedition 1955-1958 (Vol. 3): Jerusalem (Israel Program for Sci. Transl.), 2-186.

, 1971. Radiolyarii Nassellaria v planktone Mirovogo Okeana (Radiolarians of the Ocean). Issled. Fauny Morei, 9:1-294.

Popofsky, A., 1908. Die Radiolarien der Antarktis (mit Ausnahme der Tripyleen) (Radiolaria from the Antarctic [Tripylida excepted]). Dtsch. Sudpolar-Exped., 19011903, Zool., 10:183-306.

, 1913. Die Nassellarien des Warmwassergebietes (Nassellaria from warm water areas). Dtsch. Sudpolar-Exped., 1901-1903, Zool., 14:217-416.

— 1917 . Die Collosphaeriden der 1901-1903. (mit Nachtrag zu den Spumellarien und der Nassellarien). Dtsch. Südpolar-Exped., Zool., 8:235-278.

Riedel, W.R., 1958. Radiolaria in Antarctic sediments. Rep. B.A.N.Z. Antarct. Res. Exped., Ser. B, 6:217-255.

Riedel, W.R., and Sanfilippo, A., 1971. Cenozoic Radiolaria from the western tropical Pacific, Leg 7. In Winterer, E.L., Riedel, W.R., et al., Init. Repts. DSDP, 7 (Pt. 2): Washington (U.S. Govt. Printing Office), 1529-1672.

- 1978. Stratigraphy and evolution of tropical Cenozoic radiolarians. Micropaleontology, 24:61-96.

Sanfilippo, A., and Nigrini, C., 1998. Code numbers for Cenozoic low latitude radiolarian biostratigraphic zones and GPTS conversion tables. Mar. Micropaleontol., 33:109-156.

Shackleton, N.J., Baldauf, J.G., Flores, J.-A., Iwai, M., Moore, T.C., Jr., Raffi, I., and Vincent, E., 1995. Biostratigraphic summary for Leg 138. In Pisias, N.G., Mayer, L.A., Janecek, T.R., Palmer-Julson, A., and van Andel, T.H. (Eds.), Proc. ODP, Sci. Results, 138: College Station, TX (Ocean Drilling Program), 517-536.

Takahashi, K., 1991. Radiolaria: Flux, Ecology, and Taxonomy in the Pacific and Atlantic. Ocean Biocoenosis Ser., 3: Woods Hole, MA (WHOI).

Takahashi, K., and Honjo, S., 1981. Vertical flux of Radiolaria: a taxon-quantitative sediment trap study from the western tropical Atlantic. Micropaleontology, 27:140190.

Van de Paverd, P.J., 1995. Recent polycystine Radiolaria from the Snellius-II expedition [Ph.D. dissert.], Amsterdam. 
M. TESTA ET AL.

QUATERNARY RADIOLARIANS

Table T1. Geographic location and water depth of ODP Leg 180 drilled holes.

\begin{tabular}{cccc}
\hline Hole & $\begin{array}{c}\text { Latitude } \\
(\mathrm{S})\end{array}$ & $\begin{array}{c}\text { Longitude } \\
(\mathrm{E})\end{array}$ & $\begin{array}{c}\text { Water depth } \\
(\mathrm{m})\end{array}$ \\
\hline $180-$ & & & \\
$1108 \mathrm{~A}$ & $9^{\circ} 44.708^{\prime}$ & $151^{\circ} 37.514^{\prime}$ & 3162.7 \\
$1108 \mathrm{~B}$ & $9^{\circ} 44.724^{\prime}$ & $151^{\circ} 37.543^{\prime}$ & 3177.2 \\
$1109 \mathrm{~A}$ & $9^{\circ} 30.390^{\prime}$ & $151^{\circ} 34.388^{\prime}$ & 2210.9 \\
$1109 \mathrm{~B}$ & $9^{\circ} 30.396^{\prime}$ & $151^{\circ} 34.391^{\prime}$ & 2211.0 \\
$1109 \mathrm{C}$ & $9^{\circ} 30.392^{\prime}$ & $151^{\circ} 34.390^{\prime}$ & 2211.0 \\
$1109 \mathrm{D}$ & $9^{\circ} 30.380^{\prime}$ & $151^{\circ} 34.355^{\prime}$ & 2211.0 \\
$1110 \mathrm{~A}$ & $9^{\circ} 43.599^{\prime}$ & $151^{\circ} 34.511^{\prime}$ & 3246.4 \\
$1110 \mathrm{~B}$ & $9^{\circ} 43.609^{\prime}$ & $151^{\circ} 34.509^{\prime}$ & 3246.3 \\
$1110 \mathrm{C}$ & $9^{\circ} 43.599^{\prime}$ & $151^{\circ} 34.498^{\prime}$ & 3245.8 \\
$1110 \mathrm{D}$ & $9^{\circ} 43.588^{\prime}$ & $151^{\circ} 34.526^{\prime}$ & 3245.8 \\
$1111 \mathrm{~A}$ & $9^{\circ} 43.059^{\prime}$ & $151^{\circ} 34.533^{\prime}$ & 3200.7 \\
$1112 \mathrm{~A}$ & $9^{\circ} 44.749^{\prime}$ & $151^{\circ} 36.721^{\prime}$ & 3046.7 \\
$1112 \mathrm{~B}$ & $9^{\circ} 44.746^{\prime}$ & $151^{\circ} 36.714^{\prime}$ & 3046.6 \\
$1113 \mathrm{~A}$ & $9^{\circ} 45.449^{\prime}$ & $151^{\circ} 36.737^{\prime}$ & 2915.6 \\
$1114 \mathrm{~A}$ & $9^{\circ} 47.613^{\prime}$ & $151^{\circ} 34.504^{\prime}$ & 406.5 \\
$1115 \mathrm{~A}$ & $9^{\circ} 11.389^{\prime}$ & $151^{\circ} 34.450^{\prime}$ & 1149.6 \\
$1115 \mathrm{~B}$ & $9^{\circ} 11.382^{\prime}$ & $151^{\circ} 34.437^{\prime}$ & 1148.8 \\
$1115 \mathrm{C}$ & $9^{\circ} 11.383^{\prime}$ & $151^{\circ} 34.422^{\prime}$ & 1148.7 \\
$1116 \mathrm{~A}$ & $9^{\circ} 51.934^{\prime}$ & $151^{\circ} 34.508^{\prime}$ & 1851.3 \\
$1117 \mathrm{~A}$ & $9^{\circ} 46.526^{\prime}$ & $151^{\circ} 32.945^{\prime}$ & 1663.2 \\
$1117 \mathrm{~B}$ & $9^{\circ} 46.527^{\prime}$ & $151^{\circ} 32.951^{\prime}$ & 1663.2 \\
$1117 \mathrm{C}$ & $9^{\circ} 46.520^{\prime}$ & $151^{\circ} 32.943^{\prime}$ & 1663.2 \\
\hline & $9^{\circ} 35.110^{\prime}$ & $151^{\circ} 34.421^{\prime}$ & 2303.6 \\
\hline
\end{tabular}


M. TeSta et AL.

QUATERNARY RADIOLARIANS

Table T2. Full designation of the studied samples.

\begin{tabular}{cc}
\hline $\begin{array}{c}\text { Core, section, } \\
\text { interval (cm) }\end{array}$ & $\begin{array}{c}\text { Depth } \\
\text { (mbsf) }\end{array}$ \\
\hline $180-1108 \mathrm{~B}-$ & \\
$1 \mathrm{R}-1,10-12$ & 0.1 \\
$1 \mathrm{R}-2,50-52$ & 1.5 \\
$8 \mathrm{R}-1,18-20$ & 62.88 \\
$8 \mathrm{R}-1,55-57$ & 63.25 \\
$9 \mathrm{R}-1,20-22$ & 72.5 \\
$9 \mathrm{R}-1,101-103$ & 73.31 \\
$9 \mathrm{R}-1,115-117$ & 73.45 \\
$10 \mathrm{R}-1,80-82$ & 82.7 \\
$14 \mathrm{R}-1,73-75$ & 120.93 \\
$14 \mathrm{R}-2,67-69$ & 122.03 \\
$15 \mathrm{R}-2,35-37$ & 130.98 \\
$15 \mathrm{R}-2,85.5-87$ & 131.49 \\
$16 \mathrm{R}-2,30-32$ & 141.2 \\
$16 \mathrm{R}-2,104-105$ & 141.94 \\
$180-1109 \mathrm{C}-$ & \\
$2 \mathrm{H}-2,43-45$ & 9.33 \\
$2 \mathrm{H}-4,37-39$ & 12.27 \\
$3 \mathrm{H}-1,40-42$ & 17.30 \\
$3 \mathrm{H}-1,49.5-50.5$ & 17.40 \\
$3 \mathrm{H}-6,104-106$ & 25.44 \\
$4 \mathrm{H}-1,58-60$ & 26.98 \\
$4 \mathrm{H}-4,83.5-86$ & 31.74 \\
$5 \mathrm{H}-3,85-86$ & 39.75 \\
$5 \mathrm{H}-5,29-31$ & 42.19 \\
$6 \mathrm{H}-5,24-26$ & 51.64 \\
$7 \mathrm{H}-3,23-25$ & 58.13 \\
$7 \mathrm{H}-4,118-120$ & 60.58 \\
$8 \mathrm{H}-6,126-126$ & 73.16 \\
$9 \mathrm{H}-7,50-52.5$ & 83.4 \\
$10 \mathrm{H}-5,13-15$ & 89.53 \\
$11 \mathrm{H}-6,81-83$ & 101.21 \\
$12 \mathrm{X}-4,80-82$ & 107.70 \\
$12 \mathrm{X}-6,74-76$ & 110.64 \\
$13 \mathrm{X}-5,38-40$ & 118.38 \\
$14 \mathrm{X}-1,7.5-10$ & 121.68 \\
$16 \mathrm{X}-4,59-60$ & 145.89 \\
$20 \mathrm{X}-4,43-45$ & 184.23 \\
$23 \mathrm{X}-7,60-62$ & 216.35 \\
$180-1110 \mathrm{~A}-$ & \\
$1 \mathrm{H}-2,77-79$ & 2.27 \\
$1 \mathrm{H}-4,94-96$ & 5.44 \\
$2 \mathrm{H}-1,58-62$ & 7.58 \\
$2 \mathrm{H}-1,81-85$ & 7.81 \\
& \\
&
\end{tabular}

\begin{tabular}{|c|c|}
\hline $\begin{array}{l}\text { Core, section, } \\
\text { interval }(\mathrm{cm})\end{array}$ & $\begin{array}{l}\text { Depth } \\
\text { (mbsf) }\end{array}$ \\
\hline \multicolumn{2}{|l|}{ 180-1111A- } \\
\hline $8 \mathrm{R}-1,77-81$ & 68.47 \\
\hline $8 R-3,29-31$ & 70.99 \\
\hline $11 R-1,44-46$ & 97.14 \\
\hline $13 R-2,21-23$ & 117.37 \\
\hline $14 \mathrm{R}-1,60-62$ & 126.4 \\
\hline $14 R-3,62-64$ & 129.42 \\
\hline $15 R-1,38-40$ & 135.78 \\
\hline $16 \mathrm{R}-1,5-7$ & 145.05 \\
\hline $\begin{array}{l}\text { 180-1112A- } \\
\text { 1R-1, 100-101 }\end{array}$ & 1.00 \\
\hline \multicolumn{2}{|l|}{ 180-1114A- } \\
\hline 1R-CC, 23-25 & 0.23 \\
\hline $4 \mathrm{R}-1,5-7$ & 26.25 \\
\hline \multicolumn{2}{|l|}{ 180-1115A- } \\
\hline $1 \mathrm{H}-2,5-7$ & 0.33 \\
\hline $1 \mathrm{H}-4,4.5-6.5$ & 3.05 \\
\hline \multicolumn{2}{|l|}{ 180-1115B- } \\
\hline $1 \mathrm{H}-1,5-7$ & 0.05 \\
\hline 1H-1, 27-29 & 3.27 \\
\hline $2 \mathrm{H}-4,145-147$ & 13.15 \\
\hline $2 \mathrm{H}-6,130-132$ & 16.00 \\
\hline $3 \mathrm{H}-2,58-60$ & 18.78 \\
\hline $4 \mathrm{H}-4,59-62$ & 31.29 \\
\hline $4 \mathrm{H}-6,66-68$ & 34.36 \\
\hline $5 \mathrm{H}-1,19-21$ & 35.89 \\
\hline $5 \mathrm{H}-4,70-72$ & 40.9 \\
\hline $6 \mathrm{H}-2,53-55$ & 47.23 \\
\hline $6 \mathrm{H}-5,55-57$ & 51.75 \\
\hline $6 \mathrm{H}-7,39-43$ & 54.59 \\
\hline 7H-4, 77-79 & 59.97 \\
\hline $7 \mathrm{H}-6,78-80$ & 62.98 \\
\hline $8 \mathrm{H}-3,111-113$ & 68.31 \\
\hline $8 \mathrm{H}-6,17-19$ & 71.87 \\
\hline $9 \mathrm{H}-1,5-7$ & 73.75 \\
\hline $9 \mathrm{H}-3,64-66$ & 77.34 \\
\hline $10 \mathrm{H}-7,14-16.5$ & 92.34 \\
\hline $11 \mathrm{H}-3,21-23$ & 95.91 \\
\hline $11 \mathrm{H}-5,8-15$ & 98.78 \\
\hline $11 \mathrm{H}-7,9-11$ & 101.79 \\
\hline $12 \mathrm{H}-1,17-19$ & 102.37 \\
\hline $12 \mathrm{H}-4,33-35$ & 107.03 \\
\hline
\end{tabular}


Table T3. Distribution of radiolarian taxa in Holes 1108B, 1109C, 1110A, 1111A, 1112A, and 1115B. (See table note. Continued on next two pages.)

\begin{tabular}{|c|c|c|c|c|c|c|c|c|c|c|c|c|c|c|c|c|c|c|c|}
\hline \multirow{2}{*}{$\begin{array}{l}\text { Core, hole: } \\
\text { Section, interval }(\mathrm{cm}) \text { : }\end{array}$} & \multicolumn{2}{|c|}{ 180-1108B- } & \multicolumn{3}{|c|}{ 180-1109C- } & \multicolumn{3}{|c|}{ 180-1110A- } & \multicolumn{5}{|c|}{ 180-1111A- } & \multirow{2}{*}{$\begin{array}{c}\frac{180-1112 \mathrm{~A}-}{1 \mathrm{R}-1} \\
100-101\end{array}$} & \multicolumn{5}{|c|}{ 180-1115B- } \\
\hline & $\begin{array}{c}1 \mathrm{R}-1 \\
10-12\end{array}$ & $\begin{array}{c}1 \mathrm{R}-2 \\
50-52\end{array}$ & $\begin{array}{l}3 \mathrm{H}-1 \\
49-50\end{array}$ & $\begin{array}{l}4 \mathrm{H}-1 \\
58-60\end{array}$ & $\begin{array}{l}6 \mathrm{H}-5 \\
24-26\end{array}$ & $\begin{array}{l}1 \mathrm{H}-2 \\
77-79\end{array}$ & $\begin{array}{l}2 \mathrm{H}-1 \\
58-62\end{array}$ & $\begin{array}{l}2 \mathrm{H}-1 \\
81-85\end{array}$ & $\begin{array}{l}11 \mathrm{R}-1 \\
44-46\end{array}$ & $\begin{array}{l}14 \mathrm{R}-1 \\
60-62\end{array}$ & $\begin{array}{l}14 \mathrm{R}-3 \\
62-64\end{array}$ & $\begin{array}{l}15 \mathrm{R}-1 \\
38-40\end{array}$ & $\begin{array}{c}16 \mathrm{R}-1 \\
5-7\end{array}$ & & $\begin{array}{c}1 \mathrm{H}-1 \\
5-7\end{array}$ & $\begin{array}{c}2 \mathrm{H}-4 \\
145-147\end{array}$ & $\begin{array}{l}3 \mathrm{H}-2 \\
58-60\end{array}$ & $\begin{array}{l}4 \mathrm{H}-6 \\
66-68\end{array}$ & $\begin{array}{l}5 \mathrm{H}-1, \\
19-21\end{array}$ \\
\hline Acanthosphaera actinota & & & & & & & & & & & & & & & & & & & \\
\hline Acrosphaera spinosa & $\mathrm{R}$ & $\mathrm{R}$ & & & & & & $\mathrm{R}$ & $\mathrm{R}$ & $\mathrm{R}$ & $\mathrm{R}$ & $\mathrm{R}$ & & $\mathrm{R}$ & $\mathrm{R}$ & & $\mathrm{R}$ & $\mathrm{R}$ & \\
\hline Actinomma spp. & + & $\mathrm{R}$ & & + & & & & & & $\mathrm{R}$ & & & & $\mathrm{R}$ & $\mathrm{R}$ & & & $\mathrm{R}$ & + \\
\hline Actinomma boreale & & & & & & $\mathrm{R}$ & & & & & & & & & & & & & \\
\hline Actinomma haysi & & & & & & + & & & & & & & & & & & & & \\
\hline Actinomma leptoderma & + & & & & & & & & & & & & & & & & & & \\
\hline Actinomma sol & + & & & & & $\mathrm{R}$ & & & $\mathrm{R}$ & & + & + & & & & & & + & \\
\hline Amphirhopalum ypsilon & $\mathrm{R}$ & & & & & & & & & $\mathrm{R}$ & $\mathrm{R}$ & & & & + & & $\mathrm{R}$ & & \\
\hline Amphisphaera sp. & & & & & & + & & & & & & & & & & & & & \\
\hline Anthocyrtidium ophirense & $\mathrm{R}$ & & & & & & & & & & & & & & & & $\mathrm{R}$ & & \\
\hline Anthocyrtidium zanguebaricum & + & & & & & & & & + & & & & & & & & & & \\
\hline Arachnocorys umbellifera & & & & & & & & & & & & & $\mathrm{R}$ & & $\mathrm{R}$ & & & & \\
\hline Artostrobus annulatus & & & & & & & & & & & & & + & & & & & & \\
\hline Artostrobus joergenseni & & & & & & + & & & & & & & + & & & & + & & \\
\hline Botryocyrtis scutum & C & $\mathrm{F}$ & + & & & A & & C & C & $\mathrm{F}$ & C & C & $\mathrm{F}$ & $\mathrm{R}$ & $\mathrm{F}$ & + & c & + & \\
\hline Botryopyle cribrosa & + & + & & & & & & & & & & $\mathrm{R}$ & $\mathrm{R}$ & & + & & + & & \\
\hline Botryostrobus spp. & + & + & & & & & & & & & & & & $\mathrm{R}$ & & & & & \\
\hline Botryostrobus aquilonaris & + & & & & & & & & & & + & $\mathrm{R}$ & + & & & & & & \\
\hline Botryostrobus auritus/australis & $\mathrm{F}$ & & & & & $\mathrm{F}$ & & $\mathrm{R}$ & & & $\mathrm{R}$ & + & $\mathrm{R}$ & & + & & $\mathrm{F}$ & & \\
\hline Callimitra spp. & + & & & & & & & & & & & & & & & & & & \\
\hline Carpocanarium papillosum & $\mathrm{R}$ & + & & & & $\mathrm{R}$ & & $\mathrm{R}$ & + & & $\mathrm{R}$ & $\mathrm{R}$ & $\mathrm{R}$ & $\mathrm{R}$ & $\mathrm{R}$ & & $\mathrm{R}$ & & \\
\hline Carpocanistrum spp. & $\mathrm{R}$ & + & & & & $\mathrm{F}$ & & $\mathrm{F}$ & $\mathrm{F}$ & $\mathrm{R}$ & $\mathrm{F}$ & $\mathrm{R}$ & $\mathrm{F}$ & $\mathrm{R}$ & + & & $\mathrm{R}$ & & \\
\hline Cladoscenium spp. & & & & & & + & & & + & & + & & & & + & & $\mathrm{R}$ & & \\
\hline Clathrocanium coarctatum & & & & & & & & & & & & & & & + & & & & \\
\hline Clathrocanium reginae & + & & & & & & & & & & & & & & & & & & \\
\hline Collosphaera spp. & + & $\mathrm{R}$ & + & & & & & $\mathrm{F}$ & $\mathrm{F}$ & $\mathrm{R}$ & $\mathrm{R}$ & $\mathrm{R}$ & $\mathrm{R}$ & $\mathrm{R}$ & & + & & + & $\mathrm{R}$ \\
\hline Collosphaera macropora & + & & & & & & & & & & & & & & & & & & \\
\hline Collosphaera tuberosa & + & & & & & & + & & & & & & & & & & & & \\
\hline Cornutella profunda & C & $\mathrm{R}$ & & & & $\mathrm{F}$ & & $\mathrm{R}$ & $\mathrm{R}$ & & $\mathrm{R}$ & & $\mathrm{F}$ & + & $\mathrm{R}$ & & $\mathrm{R}$ & + & \\
\hline Cycladophora bicornis & $\mathrm{R}$ & + & & & & $\mathrm{F}$ & & + & $\mathrm{R}$ & $\mathrm{R}$ & & $\mathrm{R}$ & $\mathrm{R}$ & & $\mathrm{R}$ & & $\mathrm{F}$ & & \\
\hline Cycladophora davisiana cornutoides & & & & & & & & & $\mathrm{R}$ & & + & $\mathrm{R}$ & & & & & & & \\
\hline Cycladophora davisiana davisiana & & & & & & & & + & + & $\mathrm{R}$ & & $\mathrm{F}$ & $\mathrm{R}$ & & & & & & \\
\hline Cyrtolagena laguncula & + & + & & & & & & & + & & & & + & + & + & & & & \\
\hline Dictyocoryne profunda & $\mathrm{R}$ & $\mathrm{R}$ & & & & + & & $\mathrm{R}$ & $\mathrm{R}$ & + & + & & & $\mathrm{R}$ & $\mathrm{R}$ & & + & & \\
\hline Dictyocoryne truncatum & $\mathrm{F}$ & $\mathrm{R}$ & + & & & $\mathrm{R}$ & & $\mathrm{R}$ & $\mathrm{R}$ & $\mathrm{R}$ & $\mathrm{F}$ & $\mathrm{F}$ & + & $\mathrm{R}$ & $\mathrm{R}$ & & $\mathrm{F}$ & & \\
\hline Dictyophimus spp. & $\mathrm{R}$ & $\mathrm{R}$ & & & & $\mathrm{R}$ & & $\mathrm{R}$ & $\mathrm{R}$ & $\mathrm{R}$ & $\mathrm{R}$ & & $\mathrm{R}$ & $\mathrm{R}$ & $\mathrm{R}$ & + & $\mathrm{R}$ & & \\
\hline Dictyophimus crisiae & & + & & & & & & + & + & & $\mathrm{R}$ & & + & $\mathrm{R}$ & $\mathrm{R}$ & + & $\mathrm{R}$ & & \\
\hline Didymocyrtis tetrathalamus & C & $\mathrm{F}$ & + & & & $\mathrm{F}$ & & C & + & $\mathrm{R}$ & C & $\mathrm{F}$ & $\mathrm{F}$ & C & $\mathrm{F}$ & & $\mathrm{F}$ & $\mathrm{R}$ & \\
\hline Dorydruppa bensoni & & & & & & + & & & & & & & & + & & & $\mathrm{R}$ & & \\
\hline Druppatractus irregularis & & & & & & & & & & & & & + & & & & $\mathrm{R}$ & & \\
\hline Eucecryphalus gegenbauri & $\mathrm{R}$ & & & & & & & & & + & & + & $\mathrm{R}$ & & $\mathrm{R}$ & + & + & & \\
\hline Euchitonia spp. & $\mathrm{R}$ & $\mathrm{F}$ & + & & & $\mathrm{F}$ & & $\mathrm{F}$ & $\mathrm{F}$ & $\mathrm{F}$ & $\mathrm{F}$ & $\mathrm{R}$ & + & $\mathrm{F}$ & $\mathrm{R}$ & + & $\mathrm{R}$ & & \\
\hline Euchitonia elegans & + & $\mathrm{R}$ & & & & $\mathrm{R}$ & & $\mathrm{R}$ & $\mathrm{R}$ & & + & + & + & + & $\mathrm{R}$ & $\mathrm{R}$ & $\mathrm{R}$ & & \\
\hline Euchitonia furcata & $\mathrm{C}$ & $\mathrm{F}$ & & & & C & & $\mathrm{C}$ & $\mathrm{F}$ & $\mathrm{R}$ & $\mathrm{C}$ & $\mathrm{F}$ & $\mathrm{R}$ & $\mathrm{F}$ & $\mathrm{R}$ & $\mathrm{R}$ & $\mathrm{F}$ & & \\
\hline Eucyrtidium spp. & & & & & & $\mathrm{R}$ & & & & $\mathrm{R}$ & & & & & & & & & \\
\hline Eucyrtidium acuminatum & $\mathrm{R}$ & + & & & & & & & & & $\mathrm{R}$ & & $\mathrm{R}$ & & + & & + & & \\
\hline Eucyrtidium anomalum & & & & & & & & + & & & & & & & & & & & \\
\hline
\end{tabular}


Table T3 (continued).

\begin{tabular}{|c|c|c|c|c|c|c|c|c|c|c|c|c|c|c|c|c|c|c|c|c|}
\hline \multirow{2}{*}{$\begin{array}{l}\text { Core, hole: } \\
\text { Section, interval }(\mathrm{cm}) \text { : }\end{array}$} & \multicolumn{2}{|c|}{ 180-1108B- } & \multicolumn{3}{|c|}{$180-1109 \mathrm{C}-$} & \multicolumn{3}{|c|}{$180-1110 \mathrm{~A}-$} & \multicolumn{5}{|c|}{$180-1111 \mathrm{~A}-$} & \multirow{2}{*}{ 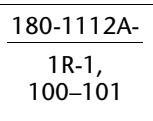 } & \multicolumn{5}{|c|}{ 180-1115B- } & \\
\hline & $\begin{array}{c}1 \mathrm{R}-1 \\
10-12\end{array}$ & $\begin{array}{l}1 \mathrm{R}-2 \\
50-52\end{array}$ & $\begin{array}{l}3 \mathrm{H}-1 \\
49-50\end{array}$ & $\begin{array}{l}4 \mathrm{H}-1 \\
58-60\end{array}$ & $\begin{array}{l}6 \mathrm{H}-5 \\
24-26\end{array}$ & $\begin{array}{l}1 \mathrm{H}-2 \\
77-79\end{array}$ & $\begin{array}{l}2 \mathrm{H}-1 \\
58-62\end{array}$ & $\begin{array}{l}2 \mathrm{H}-1 \\
81-85\end{array}$ & $\begin{array}{l}11 \mathrm{R}-1, \\
44-46\end{array}$ & $\begin{array}{l}14 \mathrm{R}-1 \\
60-62\end{array}$ & $\begin{array}{l}14 \mathrm{R}-3 \\
62-64\end{array}$ & $\begin{array}{l}15 \mathrm{R}-1 \\
38-40\end{array}$ & $\begin{array}{c}16 \mathrm{R}-1 \\
5-7\end{array}$ & & $\begin{array}{c}1 \mathrm{H}-1 \\
5-7\end{array}$ & $\begin{array}{c}2 \mathrm{H}-4 \\
145-147\end{array}$ & $\begin{array}{l}3 \mathrm{H}-2 \\
58-60\end{array}$ & $\begin{array}{l}4 \mathrm{H}-6 \\
66-68\end{array}$ & $\begin{array}{l}5 \mathrm{H}-1, \\
19-21\end{array}$ & $\sum_{2}^{\pi}$ \\
\hline Eucyrtidium hexagonatum & $\mathrm{R}$ & & & & & & & & & + & & & & & & & $\mathrm{R}$ & & & \\
\hline Eucyrtidium teuscheri teuscheri & $\mathrm{R}$ & & & & & & & & & & & & & & & & & & & \\
\hline Giraffospyris angulata & $\mathrm{R}$ & $\mathrm{R}$ & & + & & + & & $\mathrm{R}$ & $\mathrm{R}$ & & & & & & $\mathrm{R}$ & + & $\mathrm{R}$ & + & & \\
\hline Heliodiscus asteriscus & + & & & & & & & & & $\mathrm{R}$ & + & + & & & & & $\mathrm{R}$ & & & $\underline{\theta}$ \\
\hline Hexacontium spp. & $\mathrm{R}$ & & & & & $\mathrm{R}$ & & + & $\mathrm{R}$ & & & $\mathrm{R}$ & & & $\mathrm{R}$ & & $\mathrm{R}$ & & & $\underline{\underline{e}}$ \\
\hline Hexacontium enthacanthum & & & & & & & & & & & & + & $\mathrm{R}$ & & & & & & & \\
\hline Hexacontium laevigatum & + & & & & & & & & & & & & & & & & & & & 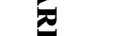 \\
\hline Hexacontium pachydermum & & & & & & & & + & & & & & & $\mathrm{R}$ & & & $\mathrm{R}$ & & & 2 \\
\hline Hexacontium pythagoraea & & & & & & + & & & & & & & & & & & & & & Z \\
\hline Hexapyle dodecantha & $\mathrm{R}$ & & & & & $\mathrm{R}$ & & + & + & $\mathrm{R}$ & $\mathrm{R}$ & $\mathrm{R}$ & & $\mathrm{R}$ & $\mathrm{R}$ & & $\mathrm{R}$ & + & & \\
\hline Hexastylus spp. & + & & & & & & & & & & & & & & & & & & & \\
\hline Hymeniastrum euclidis & $\mathrm{R}$ & + & & & & + & & $\mathrm{R}$ & + & $\mathrm{R}$ & $\mathrm{R}$ & + & & + & & + & $\mathrm{F}$ & & & \\
\hline Lamprocyclas maritalis maritalis & + & & & & & & & + & & & & & & & + & & & + & & \\
\hline Lamprocyrtis nigriniae & & & & & & & & & & & & & & + & & & & & & \\
\hline Larcoidea spp. & $\mathrm{F}$ & $\mathrm{R}$ & & & & A & & $\mathrm{F}$ & C & C & C & C & C & $\mathrm{F}$ & C & & C & & & \\
\hline Larcopyle bütschlii & $\mathrm{R}$ & + & & & & & & $\mathrm{R}$ & $\mathrm{R}$ & & + & + & & & $\mathrm{R}$ & & $\mathrm{F}$ & & & \\
\hline Larcospira quadrangula & $\mathrm{R}$ & & & & & & & + & & & & + & & & $\mathrm{R}$ & & + & & & \\
\hline Liriospyris reticulata & + & & & & & & & & & & & & & & & & & & & \\
\hline Litharachnium tentorium & + & + & & & & & & $\mathrm{R}$ & & & & $\mathrm{R}$ & $\mathrm{R}$ & & + & & + & & & \\
\hline Lithelius minor & + & & & & & & & & & & & & & & & & & & & \\
\hline Lithobotrys inflatum & $\mathrm{R}$ & & & & & & & & & & & & $\mathrm{R}$ & & & & & & & \\
\hline Lithocampe furcaspiculata & & & & & & & & & + & & & & & & & & $\mathrm{R}$ & & & \\
\hline Lithocampe platycephala & + & & & & & + & & + & & & $\mathrm{R}$ & & & & & & & & & \\
\hline Lithomelissa setosa & & & & & & + & & & & & & + & & & & & + & & & \\
\hline Lithomelissa thoracites & c & $\mathrm{R}$ & & & & $\mathrm{F}$ & & $\mathrm{F}$ & $\mathrm{R}$ & $\mathrm{R}$ & $\mathrm{F}$ & $\mathrm{F}$ & C & $\mathrm{R}$ & $\mathrm{R}$ & + & $\mathrm{F}$ & + & & \\
\hline Lophophaena bütschlii & $\mathrm{R}$ & & & & & & & $\mathrm{R}$ & & $\mathrm{R}$ & & + & $\mathrm{R}$ & & & & + & & & \\
\hline Lophophaena hispida & $\mathrm{F}$ & $\mathrm{R}$ & & & & $\mathrm{R}$ & & $\mathrm{R}$ & $\mathrm{R}$ & $\mathrm{R}$ & $\mathrm{R}$ & $\mathrm{R}$ & $\mathrm{F}$ & + & $\mathrm{R}$ & $\mathrm{R}$ & + & & & \\
\hline Lophospyris pentagona pentagona & + & & & & & & & & & & & & & & + & & & & & \\
\hline Otosphaera polymorpha & + & & & & & & & & & & & & & & & & & & & \\
\hline Peridium longispinum & + & + & & & & + & & & & & & & $\mathrm{R}$ & & + & & $\mathrm{R}$ & & & \\
\hline Peripyramis circumtexta & + & & & & & & & & & & & & & & & & & & & \\
\hline Peromelissa phalacra & $\mathrm{F}$ & & & & & $\mathrm{R}$ & & $\mathrm{R}$ & $\mathrm{R}$ & $\mathrm{R}$ & $\mathrm{R}$ & + & C & $\mathrm{R}$ & & & & & & \\
\hline Phormospyris stabilis scaphipes & + & & & & & & & & & & & & & & & & + & & & \\
\hline Phormospyris stabilis stabilis & & & & & & & & & & & & & & & + & & & & & \\
\hline Phormostichoartus corbula & + & & & & & & & & & & & + & & + & & & & & & \\
\hline Porodiscus sp. & & $\mathrm{R}$ & & & & $\mathrm{R}$ & & $\mathrm{R}$ & $\mathrm{R}$ & + & $\mathrm{R}$ & $\mathrm{R}$ & & $\mathrm{R}$ & $\mathrm{R}$ & + & $\mathrm{F}$ & & & \\
\hline Pseudodictyophimus gracilipes & & & & & & & & & & & & & $\mathrm{R}$ & & & & & & & \\
\hline Pterocanium spp. & & + & & & & & & & & & $\mathrm{R}$ & & + & & + & + & & & & \\
\hline Pterocanium praetextum praetextum & + & & & & & $\mathrm{R}$ & & $\mathrm{R}$ & & & & & & & $\mathrm{R}$ & & $\mathrm{R}$ & & & \\
\hline Pterocanium praetextum eucolpum & & & & & & & & $\mathrm{R}$ & & & & & $\mathrm{R}$ & & & & + & & & \\
\hline Pterocanium trilobum & $\mathrm{R}$ & + & & & & & & + & & & & & + & & + & & $\mathrm{R}$ & & & \\
\hline Pterocorys clausus/zancleus & $\mathrm{C}$ & $\mathrm{R}$ & & & & $\mathrm{F}$ & & $\mathrm{F}$ & $\mathrm{R}$ & $\mathrm{R}$ & $\mathrm{F}$ & $\mathrm{F}$ & $\mathrm{C}$ & $\mathrm{R}$ & C & $\mathrm{R}$ & $\mathrm{F}$ & & & \\
\hline Pterocorys hertwigii & & & & & & & & & & & & & & & + & & & & & \\
\hline Pterocorys minithorax & & & & & & & & & + & & & & & & & & + & & & \\
\hline Pylospira octopyle & + & $\mathrm{R}$ & & & & + & & $\mathrm{R}$ & $\mathrm{R}$ & $\mathrm{R}$ & $\mathrm{R}$ & $\mathrm{R}$ & & $\mathrm{R}$ & $\mathrm{R}$ & + & $\mathrm{R}$ & + & & \\
\hline Rhizoplegma boreale & & & & & & + & & & & & & & & & & & & & & \\
\hline Siphocampe arachnea & & & & & & & & + & & & & & $\mathrm{R}$ & & & & & & & \\
\hline Siphocampe lineata & & & & & & & & & $\mathrm{R}$ & & & & + & & & & & & & \\
\hline
\end{tabular}


Table T3 (continued).

\begin{tabular}{|c|c|c|c|c|c|c|c|c|c|c|c|c|c|c|c|c|c|c|c|}
\hline \multirow{2}{*}{$\begin{array}{l}\text { Core, hole: } \\
\text { Section, interval (cm): }\end{array}$} & \multicolumn{2}{|c|}{ 180-1108B- } & \multicolumn{3}{|c|}{ 180-1109C- } & \multicolumn{3}{|c|}{ 180-1110A- } & \multicolumn{5}{|c|}{ 180-1111A- } & \multirow{2}{*}{$\frac{\frac{180-1112 A-}{1 R-1}}{100-101}$} & \multicolumn{5}{|c|}{ 180-1115B- } \\
\hline & $\begin{array}{l}\text { 1R-1, } \\
10-12\end{array}$ & $\begin{array}{l}\text { 1R-2, } \\
50-52\end{array}$ & $\begin{array}{l}3 \mathrm{H}-1 \\
49-50\end{array}$ & $\begin{array}{l}4 \mathrm{H}-1 \\
58-60\end{array}$ & $\begin{array}{l}6 \mathrm{H}-5 \\
24-26\end{array}$ & $\begin{array}{l}1 \mathrm{H}-2 \\
77-79\end{array}$ & $\begin{array}{l}2 \mathrm{H}-1 \\
58-62\end{array}$ & $\begin{array}{l}2 \mathrm{H}-1 \\
81-85\end{array}$ & $\begin{array}{l}11 \mathrm{R}-1 \\
44-46\end{array}$ & $\begin{array}{l}14 \mathrm{R}-1, \\
60-62\end{array}$ & $\begin{array}{l}14 \mathrm{R}-3 \\
62-64\end{array}$ & $\begin{array}{l}15 \mathrm{R}-1 \\
38-40\end{array}$ & $\begin{array}{c}16 \mathrm{R}-1 \\
5-7\end{array}$ & & $\begin{array}{c}1 \mathrm{H}-1 \\
5-7\end{array}$ & $\begin{array}{c}2 \mathrm{H}-4 \\
145-147\end{array}$ & $\begin{array}{l}3 \mathrm{H}-2 \\
58-60\end{array}$ & $\begin{array}{l}4 \mathrm{H}-6, \\
66-68\end{array}$ & $\begin{array}{l}5 \mathrm{H}-1, \\
19-21\end{array}$ \\
\hline Siphocampe nodosaria & & & & & & $\mathrm{R}$ & & & + & & & & + & & & & & & \\
\hline Siphonosphaera polysiphonia & + & & & & & + & & + & & & + & & & & & & & & \\
\hline Spongaster tetras tetras & + & & & & & + & & $\mathrm{R}$ & $\mathrm{R}$ & $\mathrm{R}$ & $\mathrm{R}$ & + & $\mathrm{R}$ & $\mathrm{R}$ & $\mathrm{R}$ & & $\mathrm{F}$ & & \\
\hline Spongocore puella & $\mathrm{R}$ & & & & & & & + & & $\mathrm{R}$ & + & + & & + & $\mathrm{R}$ & & $\mathrm{R}$ & & \\
\hline Spongodiscus resurgens & $\mathrm{R}$ & $\mathrm{F}$ & & + & & $\mathrm{F}$ & & $\mathrm{F}$ & $\mathrm{F}$ & $\mathrm{F}$ & $\mathrm{F}$ & $\mathrm{F}$ & $\mathrm{R}$ & $\mathrm{F}$ & $\mathrm{F}$ & & C & & \\
\hline Spongopyle osculosa & $\mathrm{R}$ & + & & & & $\mathrm{R}$ & & $\mathrm{R}$ & & $\mathrm{R}$ & $\mathrm{R}$ & + & & & $\mathrm{R}$ & & + & & \\
\hline Spongotrochus glacialis & $\mathrm{F}$ & $\mathrm{R}$ & & & & $\mathrm{R}$ & & & $\mathrm{R}$ & $\mathrm{R}$ & $\mathrm{R}$ & $\mathrm{R}$ & & $\mathrm{R}$ & & & + & & \\
\hline Spongurus (?) sp. & & + & & & & + & & + & $\mathrm{R}$ & & $\mathrm{R}$ & + & & & + & & & & \\
\hline Spongurus ellipticus & & + & & & & + & & $\mathrm{R}$ & & & $\mathrm{R}$ & + & + & & & & + & & \\
\hline Spongurus pylomaticus & & & & & & & & & & & & + & & & & & + & & \\
\hline Spirocyrtis scalaris & $\mathrm{R}$ & & & & & & & & & & & & & & + & & & & \\
\hline Stylatractus spp. & $\mathrm{R}$ & $\mathrm{R}$ & & & & & & $\mathrm{R}$ & & & $\mathrm{R}$ & $\mathrm{R}$ & & & & & & & \\
\hline Stylochlamydium asteriscus & $\mathrm{F}$ & + & & + & & + & & + & + & $\mathrm{R}$ & $\mathrm{R}$ & $\mathrm{R}$ & $\mathrm{R}$ & & $\mathrm{R}$ & & $\mathrm{R}$ & & \\
\hline Stylochlamydium venustum & + & + & & & & $\mathrm{F}$ & & + & + & $\mathrm{R}$ & $\mathrm{R}$ & $\mathrm{R}$ & $\mathrm{R}$ & & $\mathrm{F}$ & & $\mathrm{F}$ & & \\
\hline Stylodictya aculeata & $\mathrm{F}$ & + & & & & $\mathrm{F}$ & & $\mathrm{R}$ & $\mathrm{F}$ & $\mathrm{F}$ & $\mathrm{F}$ & $\mathrm{R}$ & $\mathrm{R}$ & $\mathrm{R}$ & $\mathrm{R}$ & $\mathrm{R}$ & C & + & \\
\hline Stylodictya validispina & + & $\mathrm{R}$ & + & & & $\mathrm{R}$ & & $\mathrm{R}$ & $\mathrm{F}$ & $\mathrm{R}$ & C & C & $\mathrm{R}$ & $\mathrm{R}$ & $\mathrm{F}$ & $\mathrm{R}$ & $\mathrm{F}$ & $\mathrm{R}$ & \\
\hline Tessarastrum straussi & & & & & & & & & & & + & & & & & & + & & \\
\hline Tetrapyle octacantha & A & A & $\mathrm{R}$ & $\mathrm{R}$ & & A & & A & A & A & A & A & A & A & A & $\mathrm{R}$ & A & $\mathrm{R}$ & \\
\hline Theocorys veneris & + & & & & & $\mathrm{R}$ & & + & $\mathrm{R}$ & + & + & + & $\mathrm{R}$ & & $\mathrm{R}$ & & $\mathrm{R}$ & & \\
\hline Theocorythium trachelium & & & & & & & & & $\mathrm{R}$ & & + & $\mathrm{R}$ & $\mathrm{R}$ & & + & + & & & \\
\hline Theopylium tricostatum & + & & & & & & & & & & + & & + & & $\mathrm{R}$ & & & & \\
\hline Tholospyris tripodiscus & + & & & & & & & & + & & & & & & + & & & & \\
\hline Trissocyclidae spp. & $\mathrm{R}$ & $\mathrm{R}$ & & & & $\mathrm{F}$ & & $\mathrm{R}$ & $\mathrm{R}$ & $\mathrm{R}$ & $\mathrm{R}$ & + & $\mathrm{R}$ & $\mathrm{R}$ & $\mathrm{R}$ & & $\mathrm{F}$ & $\mathrm{R}$ & \\
\hline Zygocircus productus & $\mathrm{R}$ & C & + & + & + & $\mathrm{F}$ & & C & C & C & $\mathrm{R}$ & $\mathrm{F}$ & $\mathrm{F}$ & $\mathrm{F}$ & $\mathrm{F}$ & $\mathrm{F}$ & $\mathrm{F}$ & $\mathrm{R}$ & \\
\hline Spumellaria indet. & $\mathrm{F}$ & A & $\mathrm{R}$ & $\mathrm{R}$ & + & C & $\mathrm{R}$ & C & A & A & A & A & C & C & C & $\mathrm{F}$ & A & $\mathrm{R}$ & + \\
\hline Nassellaria indet. & $\mathrm{R}$ & C & + & & & $\mathrm{F}$ & + & C & C & C & C & $\mathrm{F}$ & C & C & C & $\mathrm{F}$ & C & $\mathrm{R}$ & \\
\hline
\end{tabular}

Note: $+=$ single specimen, $\mathrm{R}=$ rare $(2-5), \mathrm{F}=$ few $(6-10), \mathrm{C}=$ common $(11-25), \mathrm{A}=$ abundant $(>25)$ specimens per $27 \mathrm{~mm} \times 46 \mathrm{~mm}$ slide. 
Table T4. Radiolarian biozone assignments for the dated samples.

\begin{tabular}{|c|c|c|c|c|c|c|c|}
\hline \multirow{2}{*}{\multicolumn{2}{|c|}{$\begin{array}{c}\text { Biozones } \\
\text { (Sanfilippo and Nigrini, 1998) }\end{array}$}} & \multirow{2}{*}{$\begin{array}{c}\text { Basal Zone age } \\
\text { (Johnson et al., } \\
1989 \text { ) }\end{array}$} & \multirow{2}{*}{$\begin{array}{c}\text { Basal Zone age } \\
\text { (Caulet et al., } \\
\text { 1993) }\end{array}$} & \multirow{2}{*}{$\begin{array}{c}\text { Basal Zone age } \\
\text { (Shackleton et } \\
\text { al., 1995b) }\end{array}$} & \multirow{2}{*}{$\begin{array}{l}\frac{180-1108 \mathrm{~B}-}{1 \mathrm{R}-1} \\
10-12 \mathrm{~cm}\end{array}$} & \multicolumn{2}{|c|}{ 180-1110A- } \\
\hline & & & & & & $\begin{array}{c}1 \mathrm{H}-2 \\
77-79 \mathrm{~cm}\end{array}$ & $\begin{array}{c}2 \mathrm{H}-1 \\
58-62 \mathrm{~cm}\end{array}$ \\
\hline RN17 - Buccinosphaera invaginata & Taxon-Range Zone & 0.18 & & & & & \\
\hline RN16 - Collosphaera tuberosa & Interval Zone & & 0.42 & 0.42 & & & \\
\hline RN15 - Stylatractus universus & Concurrent Range Zone & & 0.47 & 0.61 & & & \\
\hline RN14 - Amphiropalum ypsilon & Interval Zone & & 1.10 & 1.12 & & & \\
\hline RN13 - Anthocyrtidium angulare & Interval Zone & & 1.65 & 1.74 & & & \\
\hline
\end{tabular}

Note: The biostratigraphic scheme (Sanfilippo and Nigrini, 1998) and the age assignments for the base of each biozone (Johnson et al., 1989; Caulet et al., 1993; Shackleton et al., 1995) are also shown.

Table T4 (continued).

\begin{tabular}{|c|c|c|c|c|c|c|c|c|c|c|}
\hline & & \multicolumn{5}{|c|}{ 180-1111A- } & 180-1112A- & \multicolumn{3}{|c|}{ 180-1115B- } \\
\hline \multicolumn{2}{|c|}{$\begin{array}{l}\text { Biozones } \\
\text { (Sanfilippo and Nigrini, 1998) }\end{array}$} & $\begin{array}{c}11 \mathrm{R}-1 \\
44-46 \mathrm{~cm}\end{array}$ & $\begin{array}{c}14 \mathrm{R}-1 \\
60-62 \mathrm{~cm}\end{array}$ & $\begin{array}{l}14 \mathrm{R}-3 \\
62-64 \mathrm{~cm}\end{array}$ & $\begin{array}{c}15 \mathrm{R}-1 \\
38-40 \mathrm{~cm}\end{array}$ & $\begin{array}{l}16 \mathrm{R}-1, \\
5-7 \mathrm{~cm}\end{array}$ & $\begin{array}{l}\text { 1R-1, } \\
100-101 \mathrm{~cm}\end{array}$ & $\begin{array}{l}1 \mathrm{H}-1, \\
5-7 \mathrm{~cm}\end{array}$ & $\begin{array}{l}2 \mathrm{H}-4, \\
145-147 \mathrm{~cm}\end{array}$ & $\begin{array}{c}3 \mathrm{H}-2 \\
58-60 \mathrm{~cm}\end{array}$ \\
\hline RN17 - Buccinosphaera invaginata & Taxon-Range Zone & & & & & & & & & \\
\hline RN16 - Collosphaera tuberosa & Interval Zone & & & & & & & & & \\
\hline RN15 - Stylatractus universus & Concurrent Range Zone & & & & & & & & & \\
\hline RN14 - Amphiropalum ypsilon & Interval Zone & & & & & & & & & \\
\hline RN13 - Anthocyrtidium angulare & Interval Zone & & & & & & & & & \\
\hline
\end{tabular}


M. TeSta et AL.

QUATERNARY RADIOLARIANS

Plate P1. Radiolarians from Leg 180. All are at 315×. 1. Acrosphaera spinosa (Sample 180-1108B-1R-1, 10-12 cm). 2. Amphirhopalum ypsilon (Sample 180-111A-14R-1, 60-62 cm). 3. Botryocyrtis scutum (Sample 1801110A-1H-2, 77-79 cm). 4. Cycladophora bicornis (Sample 180-1115B-3H-2, 58-60 cm). (Continued on next two pages.)

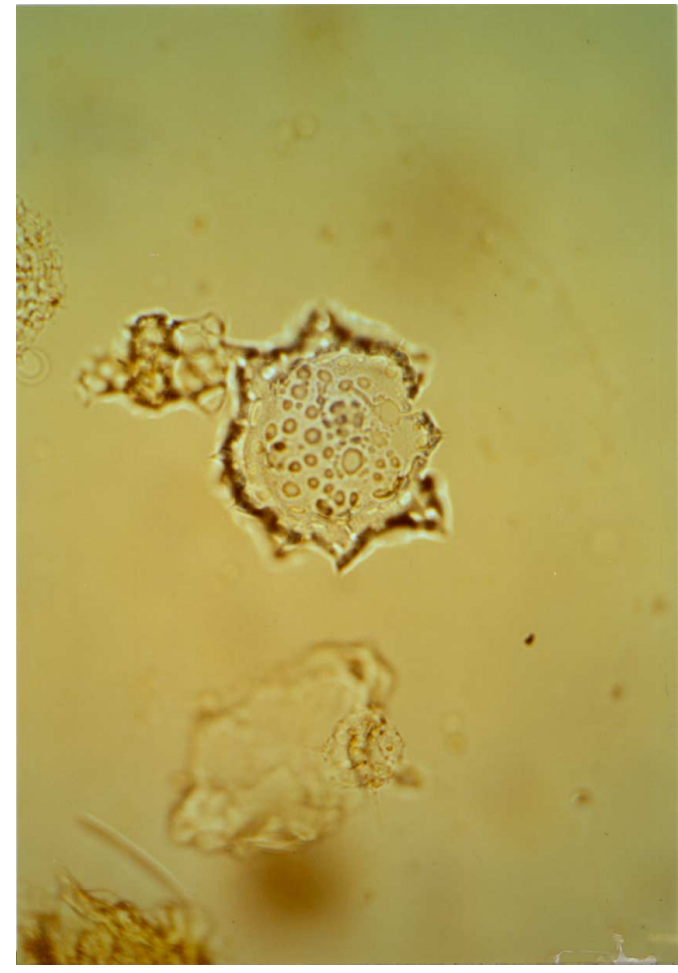

1

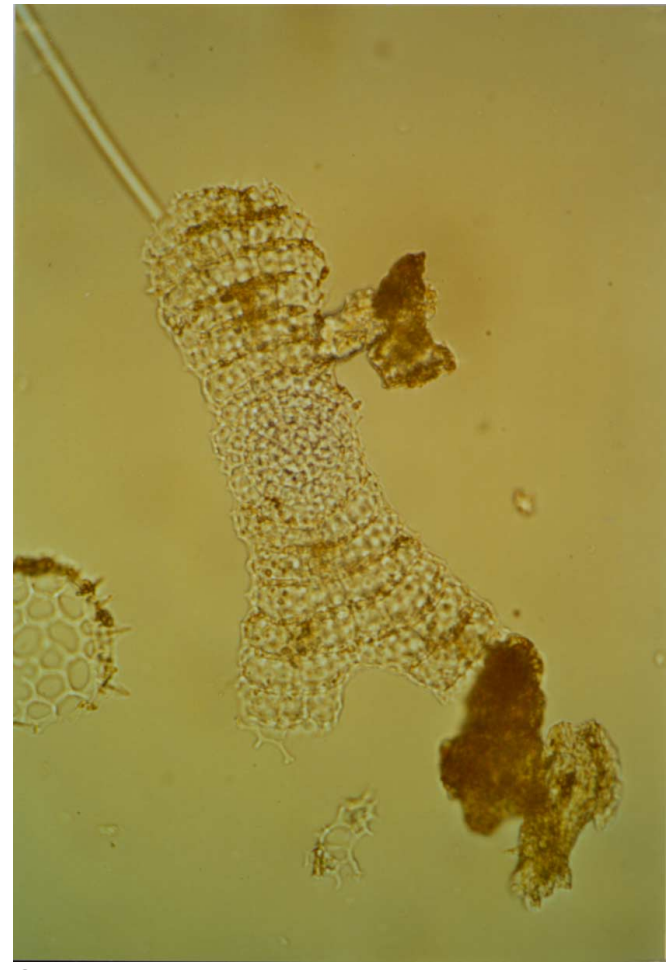

\section{2}

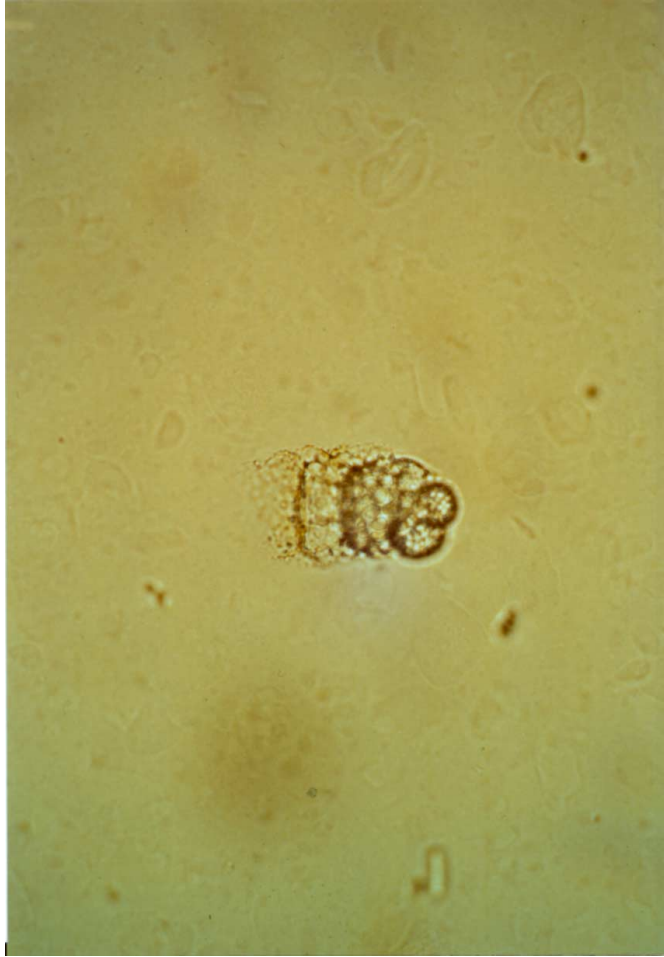

3

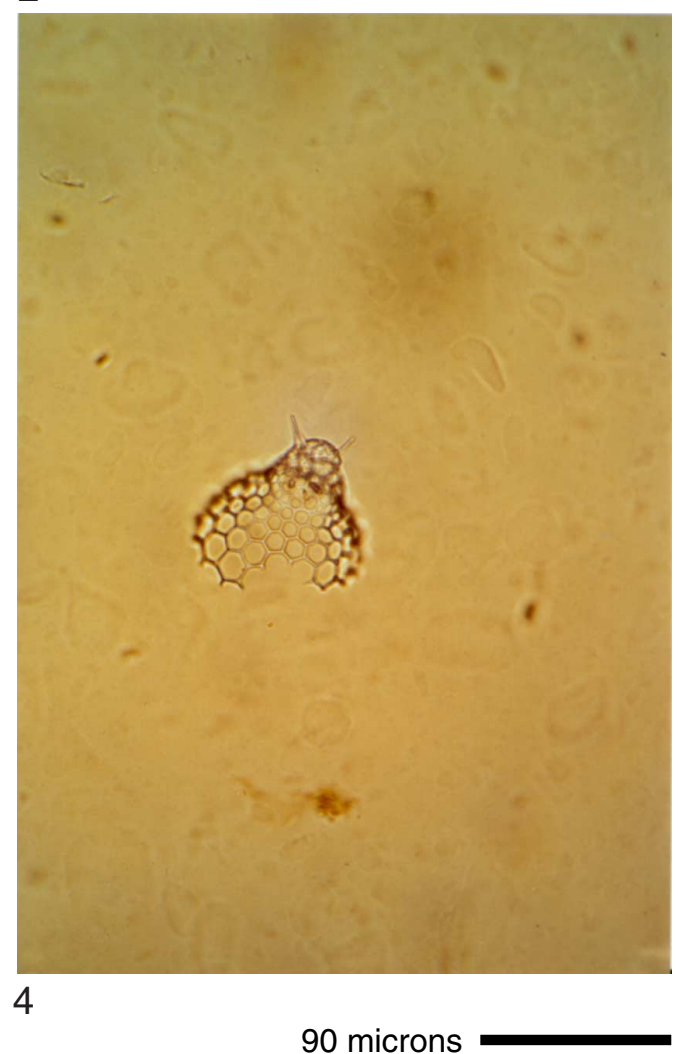


M. TeSta et AL.

QUATERNARY RADIOLARIANS

Plate P1 (continued). 5. Cornutella profunda (Sample 180-1108B-1R-1, 10-12 cm). 6. Euchitonia furcata (Sample 180-1108B-1R-2, 50-52 cm). 7. Eucyrtidium anomalum (Sample 180-1110A-2H-1, 81-85 cm). 8. Lamprocyclas maritalis maritalis (Sample 180-1108B-1R-1, 10-12 cm).

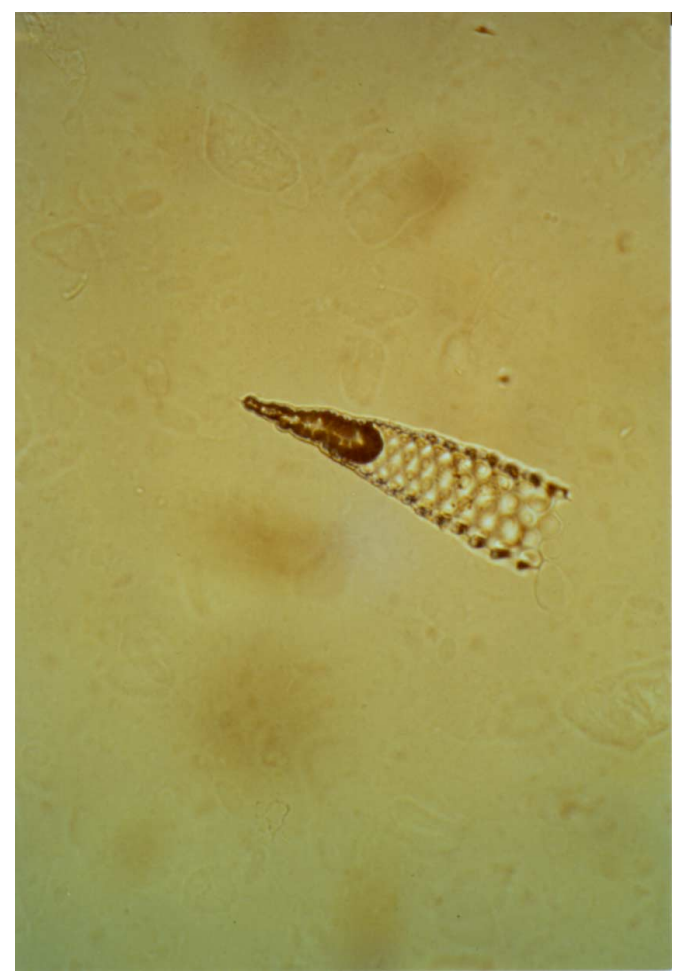

5
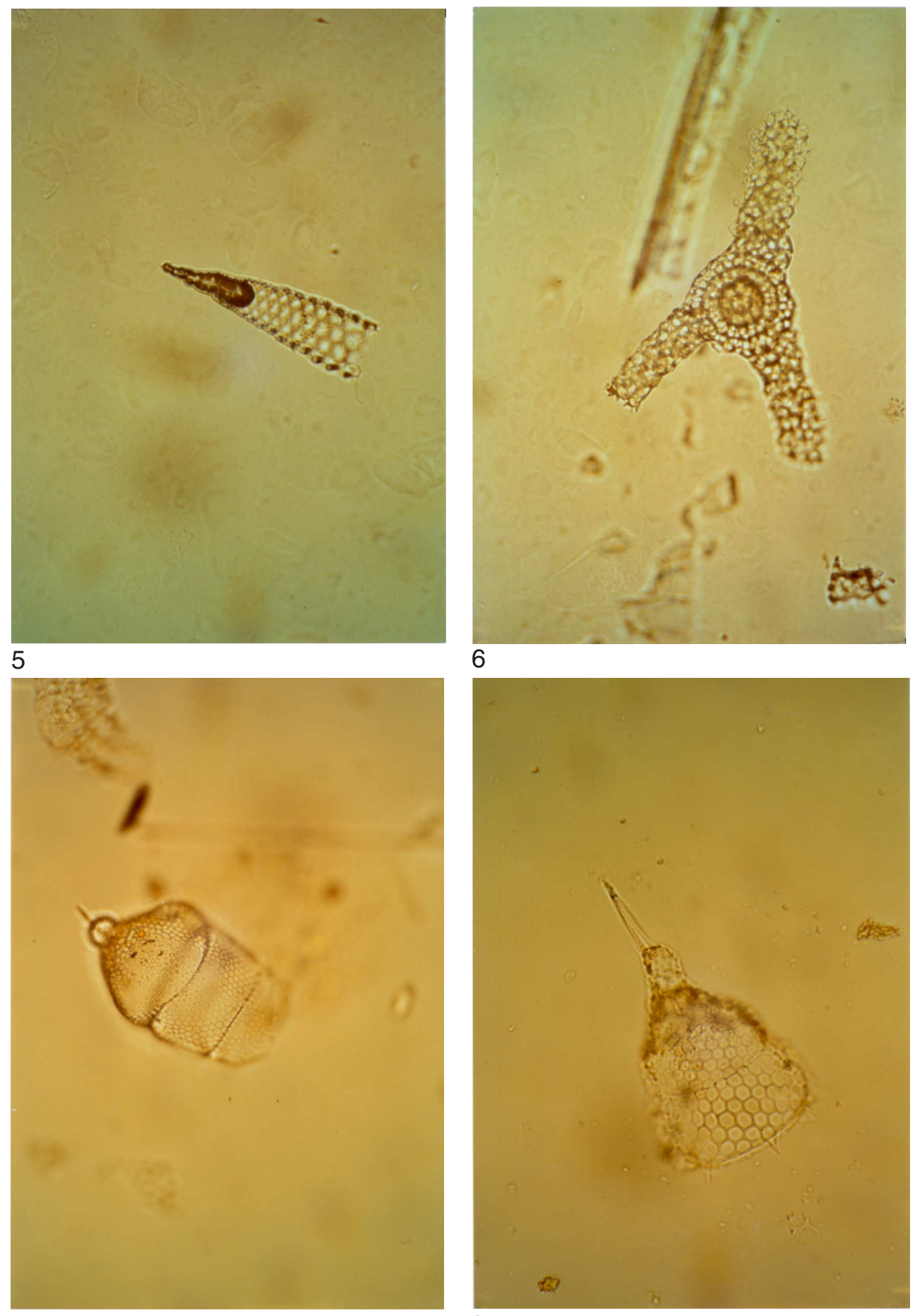

7

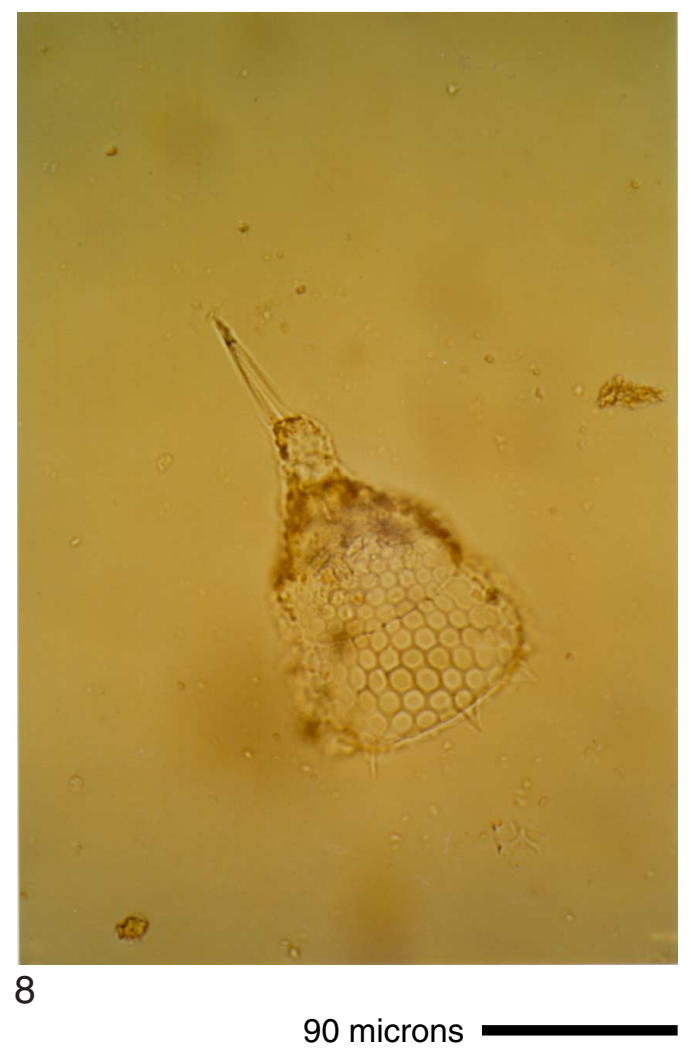


M. TeSta et AL.

QUATERNARY RADIOLARIANS

Plate P1 (continued). 9. Pterocanium praetextum praetextum (Sample 180-1115B-1H-1, 5-7 cm). 10. Spongaster tetras tetras (Sample 180-1111A-1R-1, 44-46 cm). 11. Spongocore puella (Sample 180-1108B-1R-1, 10$12 \mathrm{~cm}$ ). 12. Spongopyle osculosa (Sample 180-1110A-1H-2, 77-79 cm).

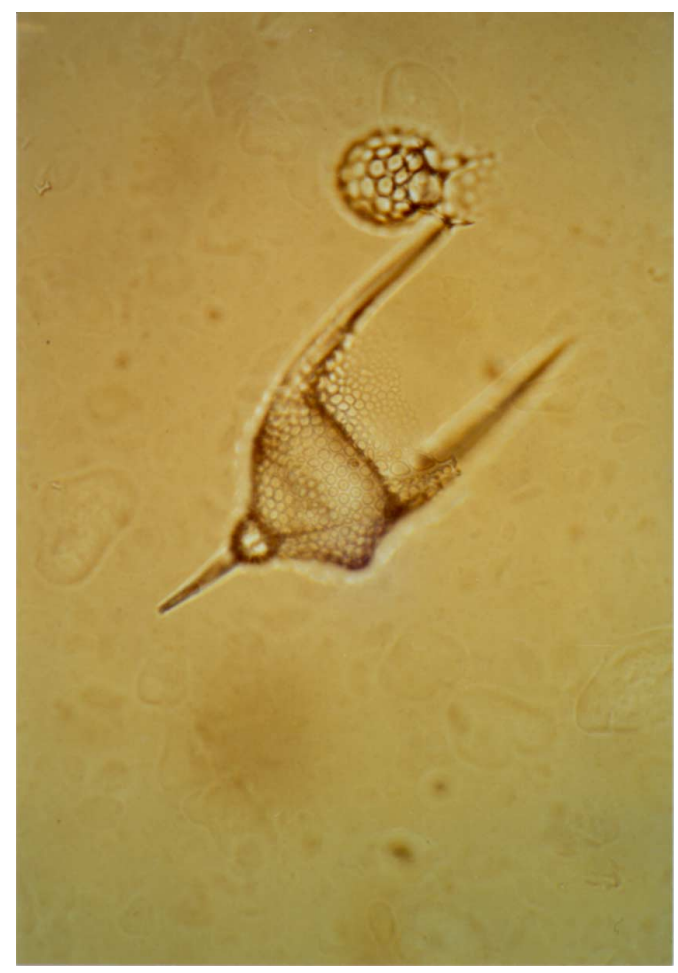

9

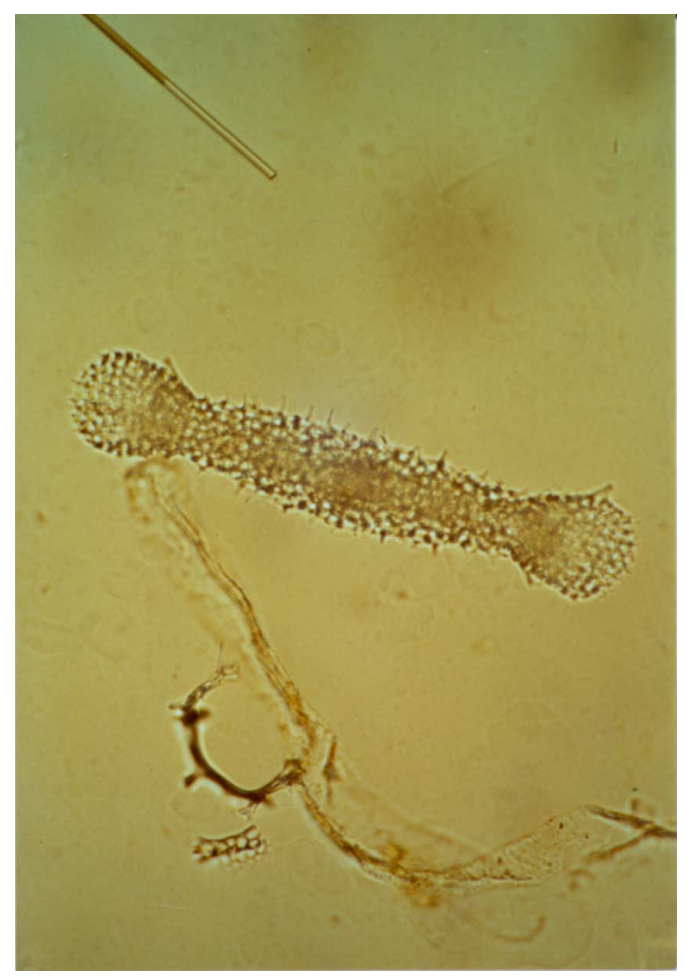

11

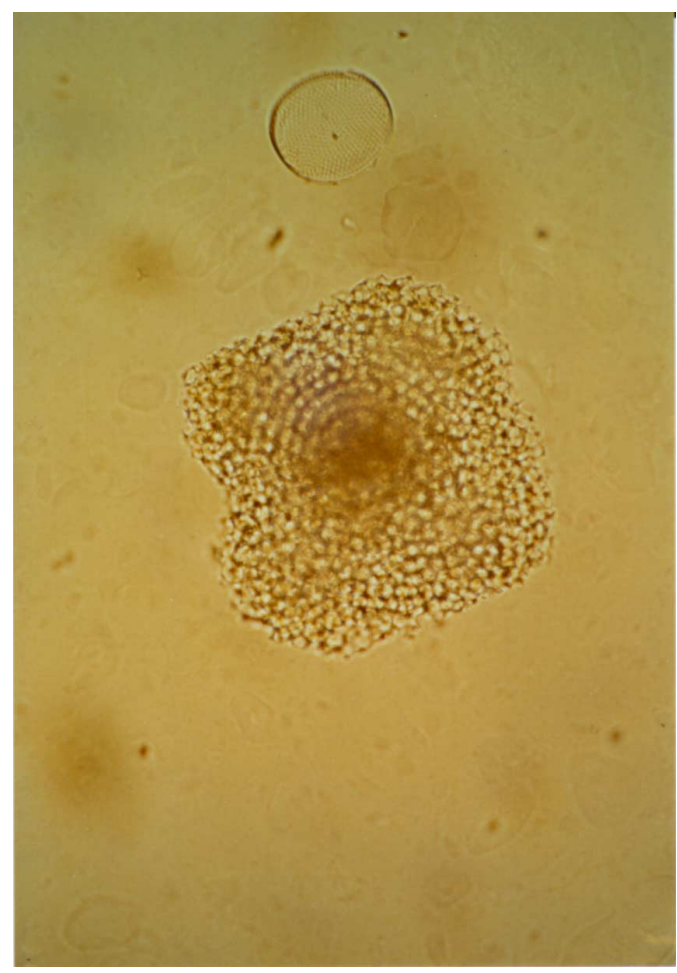

10

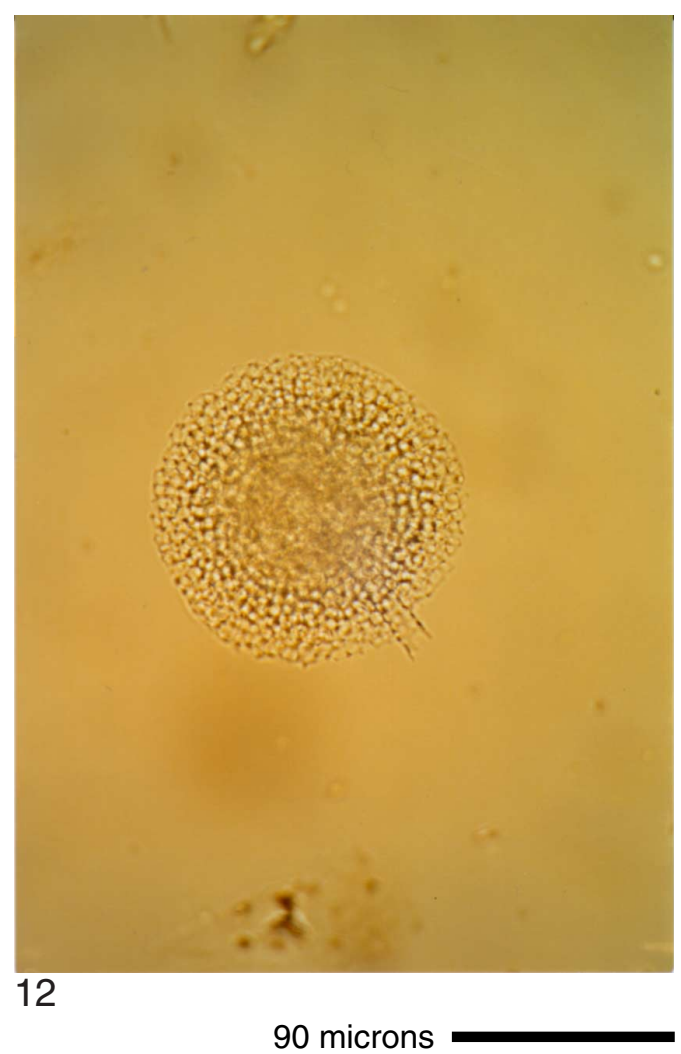

